\title{
Towards a Comprehensive Model of Jet Noise using an Acoustic Analogy and Steady RANS Solutions
}

\author{
Steven A. E. Miller* \\ The National Aeronautics and Space Administration
}

\begin{abstract}
An acoustic analogy is developed to predict the noise from jet flows. It contains two source models that independently predict the noise from turbulence and shock wave shear layer interactions. The acoustic analogy is based on the Euler equations and separates the sources from propagation. Propagation effects are taken into account by calculating the vector Green's function of the linearized Euler equations. The sources are modeled following the work of Tam and Auriault, Morris and Boluriaan, and Morris and Miller. A statistical model of the two-point cross-correlation of the velocity fluctuations is used to describe the turbulence. The acoustic analogy attempts to take into account the correct scaling of the sources for a wide range of nozzle pressure and temperature ratios. It does not make assumptions regarding fine- or large-scale turbulent noise sources, selfor shear-noise, or convective amplification. The acoustic analogy is partially informed by three-dimensional steady Reynolds-Averaged Navier-Stokes solutions that include the nozzle geometry. The predictions are compared with experiments of jets operating subsonically through supersonically and at unheated and heated temperatures. Predictions generally capture the scaling of both mixing noise and BBSAN for the conditions examined, but some discrepancies remain that are due to the accuracy of the steady RANS turbulence model closure, the equivalent sources, and the use of a simplified vector Green's function solver of the linearized Euler equations.
\end{abstract}

\section{Nomenclature}

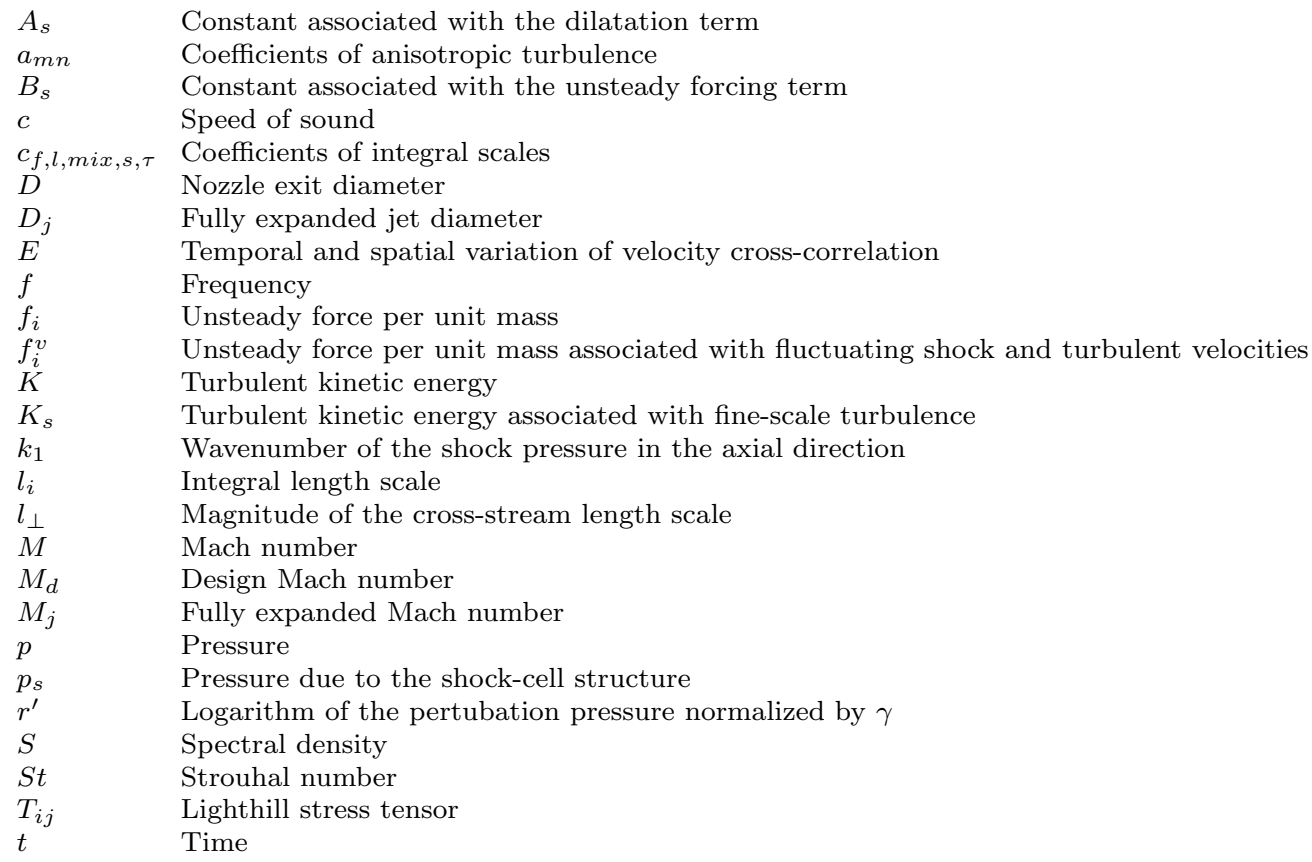

${ }^{*}$ Research Aerospace Engineer, Aeroacoustics Branch, NASA Langley Research Center, 2 N. Dryden St. MS 461, Hampton, VA, 23681, USA, AIAA Member, s.miller@nasa.gov 


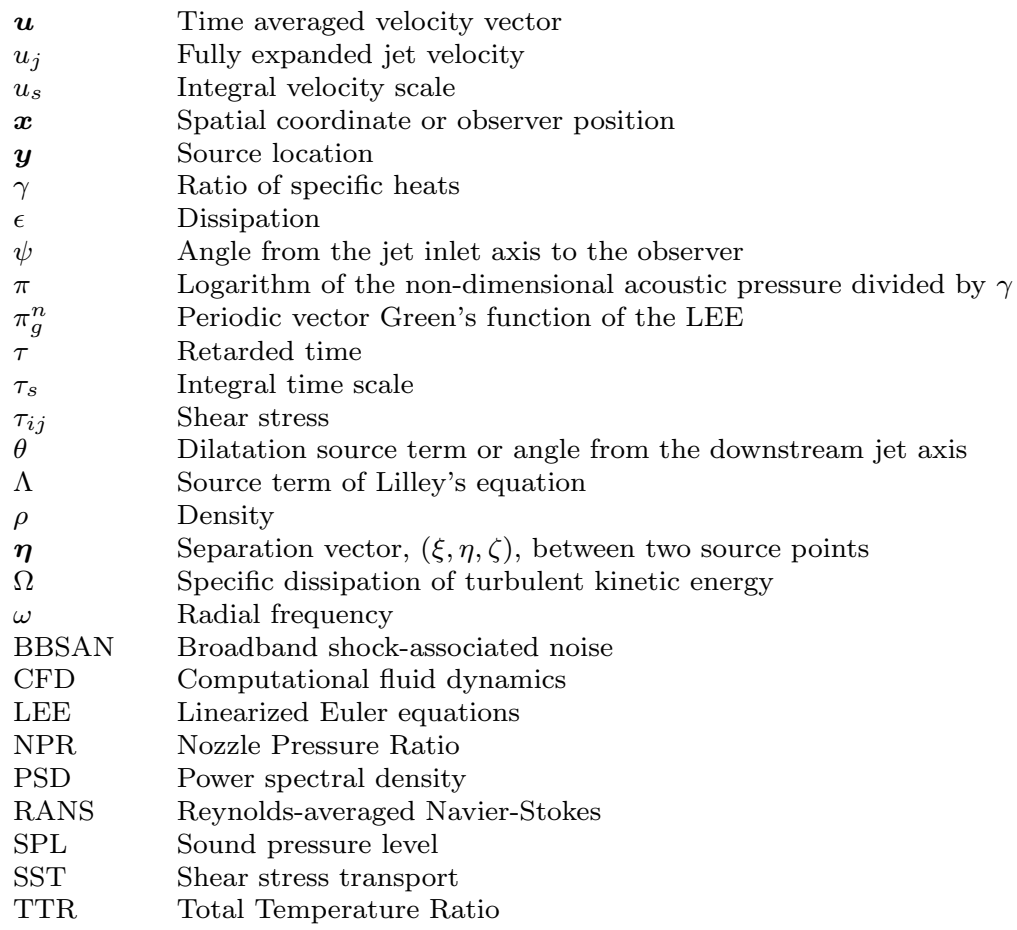

\section{Introduction}

This paper presents an acoustic analogy for jet noise that is based on the Euler equations. The model contains equivalent sources for the turbulent mixing noise and the broadband shock-associated noise (BBSAN). The comprehensive model development is based on two separate acoustic analogies, one for the mixing noise and the other for the BBSAN. The evaluation of the comprehensive analogy or the summation of the spectral density of the two independent analogies results in a prediction for total jet noise. These models are designed for a wide range of Mach numbers and temperature ratios. In the context of this theory there are no assumptions of shear-, self-, large-scale, or fine-scale noise sources or spectra.

The acoustic analogy for turbulent mixing noise is inspired by the work of Morris and Farassat, ${ }^{1}$ Morris and Boluriaan, ${ }^{2}$ Tam and Auriault ${ }^{3}$ and physical measurements. The equivalent sources of the acoustic analogy consist of the dilatation and unsteady forces per unit mass. The dilatation term is consistent with that of Tam and Auriault ${ }^{3}$ and the unsteady forces per unit mass are equivalent to those of Morris and Boluriaan. ${ }^{2}$ These choices of terms allow for the acoustic intensity to scale with increasing jet Mach number and temperature. The acoustic analogy for BBSAN is the model of Morris and Miller. ${ }^{4}$ A statistical model of the two-point cross-correlation of the turbulent velocity fluctuations describes the properties of the turbulence. Frequency dependence of the integral length scale is based on the work of Lieb and Goldstein ${ }^{5}$ and Morris and Boluriaan. ${ }^{2}$

The models are dependent on the vector Green's function of the linearized Euler equations (LEE) and a steady Reynolds-Averaged Navier-Stokes (RANS) solution. Steady RANS represents a compressible base flow of the LEE. The computational domain contains the complete nozzle geometry and fully resolves the jet plume. Steady RANS solutions are performed with the NASA Langley Fully-Unstructured Navier-Stokes (FUN3D) Computational Fluid Dynamics (CFD) package. The numerical solutions depend on the closure by the Menter ${ }^{6}$ Shear Stress Transport (SST) turbulence model, the nozzle geometry, and boundary conditions. Additional details regarding the solution methodology and computational domains are discussed in a later section. Predictions are then compared with measured results.

\section{Characteristics of Jet Noise}

Figure 1 shows normalized power spectral densities (PSD) of pressure from a supersonic off-design jet in the far-field, in which the dominant noise types are labeled. The $x$-axis represents non-dimensional frequency in terms of Strouhal number, $S t=f D_{j} / u_{j}$, where $f$ is the frequency, $u_{j}$ is the fully expanded jet velocity, and 
$D_{j}$ is the fully expanded diameter. The $y$-axis represents lossless Sound Pressure Level (SPL) per unit $S t$, which is PSD plus $10 \log _{10}\left[u_{j} / D_{j}\right] . \psi$ is the angle from the jet upstream axis to the observer. Mixing noise, labeled in Fig. 1, is induced by the turbulent mixing in the jet, and is dominant in the downstream direction of the jet flow. BBSAN is labeled in Fig. 1 and is dominant at the sideline and upstream direction at mid to high frequencies. Discrete components can sometimes be observed in the spectra if the jet is operating off-design and are called screech.

Mixing noise occurs at all jet Mach numbers and temperature regimes. At sufficiently high jet Mach numbers the directivity resembles a cardioid. The sound directivity is due to a combination of the nature of the source and the sound propagation through the jet itself. Tam ${ }^{7}$ showed how turbulence contributes to far-field jet noise by differentiating the source with fine- and large-scale noise similarity spectra. Jet noise has been reviewed by many investigators, including Ffowcs Williams ${ }^{8,9}$ and Goldstein, ${ }^{10}$ and specifically for supersonic jet noise, by Tam. ${ }^{11}$ When the convective Mach number of the jet is high enough another mixing noise mechanism is enabled that is called Mach wave radiation. For an overview of Mach wave radiation see Tam. $^{12}$

A pressure mismatch between the jet flow and the ambient environment creates a shock cell structure as shown in the schlieren of Fig 2. Shock-associated noise is divided into two parts called screech and BBSAN. Screech, first observed and described by Powell, ${ }^{13}$ consists of discrete tones and is not always observed in the far-field PSD. It is caused by a feedback loop consisting of acoustic waves propagating upstream due to their reflection by shock waves and consequently being reflected downstream by the airframe or nozzle. For more information on screech see the comprehensive review by Raman. ${ }^{14}$ BBSAN consists of multiple broad lobes and is dominant over a wide range of frequencies in the sideline and upstream directions relative to the jet flow. It occurs due to large-scale coherent turbulence interacting with the shock waves in the shear layer. The resultant acoustic waves combine constructively and destructively at observer locations to create the broad lobes. It was first characterized experimentally and modeled by Harper-Bourne and Fisher. ${ }^{15}$ Notable models for the prediction of BBSAN are by Harper-Bourne and Fisher, ${ }^{15}$ Tam, ${ }^{16}$ Tam, ${ }^{17}$ and Morris and Miller. ${ }^{4}$

\section{Previous Approaches and their Relation to the Present}

Lighthill, in his now famous works on general theory ${ }^{18}$ and turbulence as a source of sound, ${ }^{19}$ introduced the acoustic analogy. By combining the time derivative of the continuity equation with the divergence of the momentum equation, an inhomogeneous wave equation results,

$$
\frac{\partial^{2} \rho}{\partial t^{2}}-c_{\infty}^{2} \frac{\partial^{2} \rho}{\partial x_{i} \partial x_{i}}=\frac{\partial^{2} T_{i j}}{\partial x_{i} \partial x_{j}}
$$

where $T_{i j}=\rho u_{i} u_{j}-\tau_{i j}+\left(p-\rho c_{\infty}^{2}\right) \delta_{i j}$ is the Lighthill stress tensor. A solution for $\rho$ can immediately be formed by the convolution integral of the Green's function of the wave equation with the double divergence of $T_{i j}$. Lighthill constructed a model for $T_{i j}$ consisting of convecting quadruples that resulted in an estimate of jet noise intensity scaling as $M_{j}^{8}$.

Proudman, ${ }^{20}$ created an expression for the acoustic power from isotropic turbulence using both dimensional analysis and the acoustic analogy approach of Lighthill. ${ }^{18}$ Proudman showed that the acoustic power per unit mass from isotropic turbulence is proportional to $\alpha \epsilon M^{5}$, where $\epsilon$ is the dissipation, $M$ is the Mach number based on turbulent velocity fluctuations, and $\alpha$ is a constant determined from the statistical theory of Heisenberg. ${ }^{21}$

Curle $^{22}$ examined the effects of noise from surfaces by extending Lighthill's ${ }^{18}$ theory. Later, Ffowcs Williams and Hawkings ${ }^{23}$ constructed generalized forms of the continuity and momentum equations involving two regions of the flow-field and created an acoustic analogy that involved the generalized density perturbation, a generalized Lighthill stress tensor, and two additional source terms that governed the sound from turbulence and the effect of moving surfaces. The latter two terms have characteristics associated with traditional acoustic dipole and monopole theory.

Perhaps the most important development in the field of aeroacoustics after the work of Lighthill ${ }^{18,19}$ was by Lilley ${ }^{24}$ who created what is now known as Lilley's equation. Lilley's equation is,

$$
\frac{\bar{D}^{3} r^{\prime}}{D t^{3}}-\frac{\bar{D}}{D t}\left(\bar{c}^{2} \nabla^{2} r^{\prime}\right)-\frac{\partial \bar{c}^{2}}{\partial y_{2}} \frac{\bar{D}}{D t} \frac{\partial r^{\prime}}{\partial y_{2}}+2 \bar{c}^{2} \frac{\partial \bar{u}_{1}}{\partial y_{2}} \frac{\partial^{2} r^{\prime}}{\partial y_{1} \partial y_{2}}=\Lambda(\boldsymbol{y}, t)
$$


where $r^{\prime}=(1 / \gamma) \ln p, \bar{D} / D t=\partial / \partial t+u_{1} \partial / \partial x_{1}$, and $\bar{u}_{i}$ and $\bar{c}$ are the mean velocity and speed of sound respectively. $\Lambda$ is a nonlinear source term that involves the base flow and perturbation quantities and doesn't contain $r^{\prime}$. This represents a major advantage over Lighthill's approach. Also, the operator contains gradient terms involving the averaged velocity and mean enthalpy. This point is stressed as the effects of sound refraction and propagation due to the jet shear layer are taken into account directly and are separated explicitly from the source model. Unlike the approaches of Lighthill, Proudman, or Curle, we follow Lilley's approach in the spirit of not having dependent variables on the right hand side of our equations when the acoustic analogy is formed and include refraction effects in the operator.

Khavaran ${ }^{25}$ used a prediction methodology that followed the 'MGB' code of Mani et al. ${ }^{26}$ but differed significantly by informing the equivalent sources with a steady RANS solution. This is unlike the approach of Mani et al. ${ }^{26}$ which calculated the jet flow following the approach of Reichardt. ${ }^{27}$ The approach of Khavaran ${ }^{25}$ has the benefit of including the nozzle geometry in the calculation and shock waves within the jet plume. In this work we follow Khavaran ${ }^{25}$ by including the nozzle geometry and shock waves with steady RANS solutions.

Goldstein $^{28}$ showed that the Navier-Stokes equations can be rearranged so that the right hand side consists of sources that are dependent on generalized external stresses and energy flux perturbations. The left hand side operator consists of the linearized Navier-Stokes equations. The strength of the approach can be demonstrated by using different base flows. As an example, a parallel flow assumption for the base flow results in the inhomogeneous Pridmore-Brown equation (like Lilley's ${ }^{24}$ equation).

Tam and Auriault ${ }^{3}$ developed a mathematical theory to predict the noise from fine-scale turbulence based on the premise that jet mixing noise consists of two distinct spectra: fine-scale and large-scale, based on experimental observation of mixing noise developed by Tam et al. ${ }^{7}$ Tam and Auriault ${ }^{3}$ used a gas kinetic theory to relate the time rate of change of the turbulent kinetic energy of the fine-scale turbulence per unit mass in the moving frame to the source strength. They argued that the acoustic pressure is related to the turbulent kinetic energy of the fine-scale turbulence as, $p=(1 / 3) \rho<\boldsymbol{u}^{2}>=(2 / 3) \rho K_{s}$, where $u$ are the fluctuating quantities of velocity and $K_{s}$ is the turbulent kinetic energy of the fine-scale turbulence. The governing equations are the LEE with the right hand source of the momentum equation equal to $-\partial q_{s} / \partial x_{i}$. A closed form solution for the acoustic pressure is found by the convolution integral of the vector Green's function of the LEE with the source term. The predictions of the fine-scale mixing noise agree very well with measurement and scale correctly with both jet velocity and temperature. We elect to use a source term for the fluid dilation that is equivalent to Tam and Auriault. ${ }^{3}$

Morris and Farassat ${ }^{1}$ compared the acoustic analogy approach of Lighthill, ${ }^{18}$ the model for fine-scale turbulence of Tam and Auriault, ${ }^{3}$ and a modified form of Lighthill's approach based on the same assumptions of Tam and Auriault. It was shown that with consistent descriptions of the statistics of turbulence within the jet plume, equivalent prediction formulas at the sideline direction of the jet can be formed based on the approach of the acoustic analogy (or Tam and Auriault). The work of Morris and Farassat ${ }^{1}$ inspired an acoustic analogy to be developed by Morris and Boluriaan ${ }^{2}$ that is based on the Euler equations, and consists of the acoustic pressure written in terms of its logarithm (following Lilley ${ }^{24}$ ) and the acoustic velocity, but retains viscosity when determining the turbulent mean flow. A left hand side operator of the LEE is formed with equivalent sources of the dilatation and an unsteady force per unit mass. The approach within this paper draws heavily upon Morris and Boluriaan by using the same source term for the unsteady forces per unit mass and retains the advantage of formulating the operator of the LEE with only four unknowns.

Harper-Bourne and Fisher ${ }^{15}$ proposed that BBSAN depends on the coherent interaction of turbulence in the jet shear layer with the nearly periodic shock cell structure. This is modeled as a series of correlated point sources that radiate constructively or destructively. The prediction scheme depends on knowledge of the rate of decay of the turbulence correlation between shocks, as well as the characteristic spectral shape of the radiated noise generated by each interaction. Tam ${ }^{16}$ developed a stochastic methodology for the prediction of BBSAN. It depends on the large-scale turbulence of the jet shear layer being modeled as a superposition of random instability waves interacting with the nearly periodic shock cell structure. This interaction yields an interference pattern of traveling waves with phase velocities higher than the speed of sound. The instability waves give rise to noise radiation at large angles from the jet downstream axis. Tam $^{17}$ modified the method of Tam ${ }^{16}$ to include moderately heated jets and additional corrections for overor under-expanded jets. Neither of the approaches of Harper-Bourne and Fisher ${ }^{15}$ or $\operatorname{Tam}^{16}$ are based on the acoustic analogy and they are incompatible mathematically with the previously discussed approaches. Morris and Miller ${ }^{29}$ developed an acoustic analogy for BBSAN based on the Euler equations, and it drew 
heavily on the work of Harper-Bourne and Fisher ${ }^{15}$ and Tam and Auriault. ${ }^{3}$ Their approach is used here as it is compatible with the current mathematical formulations with minimal modification.

\section{Mathematical Theory}

The acoustic analogy presented herein is based on that of Morris and Farassat. ${ }^{1}$ The governing equations are the Euler equations,

$$
\begin{gathered}
\frac{D \pi}{D t}+\frac{\partial u_{i}}{\partial x_{i}}=0 \\
\frac{D u_{i}}{D t}+c^{2} \frac{\partial \pi}{\partial x_{i}}=0
\end{gathered}
$$

where $D / D t$ is the material derivative and $\pi=\gamma^{-1} \ln \left(p / p_{\infty}\right)$. By linearizing the governing equations about a mean flow and retaining terms on the left hand side that are linear in fluctuation, then the inhomogeneous LEE are formed.

$$
\frac{\partial \pi^{\prime}}{\partial t}+\bar{u}_{j} \frac{\partial \pi^{\prime}}{\partial x_{j}}+\frac{\partial u_{i}^{\prime}}{\partial x_{i}}=\theta
$$

and,

$$
\frac{\partial u_{i}^{\prime}}{\partial t}+\bar{u}_{j} \frac{\partial u_{i}^{\prime}}{\partial x_{j}}+u_{j}^{\prime} \frac{\partial \bar{u}_{i}}{\partial x_{j}}+\bar{c}^{2} \frac{\partial \pi}{\partial x_{i}}=f_{i}
$$

where $\theta$ is the dilatation rate and $f_{i}$ is the unsteady force per unit mass. In both the equivalent sources only the second order fluctuations are considered to contribute to the sound field. Note that $\pi^{\prime} \simeq \gamma^{-1} \ln (1+$ $\left.p^{\prime} / p_{\infty}\right) \simeq \gamma^{-1} p^{\prime} / p_{\infty}$. The vector Green's function of the LEE is defined as,

$$
\frac{D_{o} \pi_{g}^{n}}{D t}+\frac{\partial u_{g i}^{n}}{\partial x_{i}}=\delta(\boldsymbol{x}-\boldsymbol{y}) \delta(t-\tau) \delta_{0 n}
$$

and,

$$
\frac{D_{o} u_{g i}^{n}}{D t}+u_{g j}^{n} \frac{\partial \bar{u}_{i}}{\partial x_{j}}+\bar{c}^{2} \frac{\partial \pi_{g}^{n}}{\partial x_{i}}=\delta(\boldsymbol{x}-\boldsymbol{y}) \delta(t-\tau) \delta_{i n},
$$

where $D_{o}$ is the material derivative about the base flow. Let the vector Green's function be periodic,

$$
\begin{aligned}
\pi_{g}^{n}(\boldsymbol{x}, t \mid \boldsymbol{y}, \tau) & =\frac{1}{2 \pi} \int_{-\infty}^{\infty} \pi_{g}^{n}(\boldsymbol{x} \mid \boldsymbol{y} ; \omega) \exp [i \omega(t-\tau)] d \omega \\
\pi_{g}^{n}(\boldsymbol{x} \mid \boldsymbol{y} ; \omega) & =\int_{-\infty}^{\infty} \pi_{g}^{n}(\boldsymbol{x}, t \mid \boldsymbol{y}, \tau) \exp [-i \omega(t-\tau)] d \tau
\end{aligned}
$$

It is now simple to write the fluctuating far-field pressure as a convolution integral of the vector Green's function and the equivalent sources,

$$
p^{\prime}(\boldsymbol{x}, t)=\gamma p_{\infty} \int_{-\infty}^{\infty} \cdots \int_{-\infty}^{\infty} \pi_{g}^{0}(\boldsymbol{x}, t \mid \boldsymbol{y}, \tau) \theta(\boldsymbol{y}, \tau)+\sum_{n=1}^{3} \pi_{g}^{n}(\boldsymbol{x}, t \mid \boldsymbol{y}, \tau) f_{n}(\boldsymbol{y}, \tau) d \tau d \boldsymbol{y}
$$

The spectral density is defined as the Fourier transform of the autocorrelation of the fluctuating pressure,

$$
S(\boldsymbol{x}, \omega)=\int_{-\infty}^{\infty} \overline{p^{\prime}(\boldsymbol{x}, t) p^{\prime}(\boldsymbol{x}, t+\tau)} \exp [-i \omega \tau] d \tau .
$$

Equations 11 and 12 yield, 


$$
\begin{aligned}
& S(\boldsymbol{x}, \omega)=\rho_{\infty}^{2} c_{\infty}^{4} \int_{-\infty}^{\infty} \ldots \int_{-\infty}^{\infty}\left\{\pi_{g}^{0}(\boldsymbol{x}, \boldsymbol{y},-\omega) \pi_{g}^{0}(\boldsymbol{x}, \boldsymbol{y}+\boldsymbol{\eta}, \omega) \overline{\theta(\boldsymbol{y}, \tau) \theta(\boldsymbol{y}+\boldsymbol{\eta}, t+\tau)}\right. \\
& \left.+\sum_{n=1}^{3} \sum_{m=1}^{3} \pi_{g}^{n}(\boldsymbol{x}, \boldsymbol{y},-\omega) \pi_{g}^{m}(\boldsymbol{x}, \boldsymbol{y}+\boldsymbol{\eta}, \omega) \overline{f_{n}(\boldsymbol{y}, \tau) f_{m}(\boldsymbol{y}+\boldsymbol{\eta}, t+\tau)}\right\} \exp [-i \omega \tau] d \tau d \boldsymbol{\eta} d \boldsymbol{y} .
\end{aligned}
$$

By definition the periodic vector Green's function of the LEE is,

$$
\pi_{g}^{n *}(\boldsymbol{x}, \boldsymbol{y}, \omega)=\pi_{g}^{n}(\boldsymbol{x}, \boldsymbol{y},-\omega)
$$

and assuming that the observer is in the far-field relative to the source, we relate two closely placed source points in the jet,

$$
\pi_{g}^{m}(\boldsymbol{x}, \boldsymbol{y}+\boldsymbol{\eta}, \omega) \simeq \pi_{g}^{m}(\boldsymbol{x}, \boldsymbol{y}, \omega) \exp \left[\frac{-i \omega}{c_{\infty} x}\left(x_{1} \xi+x_{2} \eta+x_{3} \zeta\right)\right] .
$$

Using Eqns. 14 and 15 with Eqn. 13 yields,

$$
\begin{array}{r}
S(\boldsymbol{x}, \omega)=\rho_{\infty}^{2} c_{\infty}^{4} \int_{-\infty}^{\infty} \ldots \int_{-\infty}^{\infty}\left\{\pi_{g}^{* 0}(\boldsymbol{x}, \boldsymbol{y}, \omega) \pi_{g}^{0}(\boldsymbol{x}, \boldsymbol{y}, \omega) \overline{\theta(\boldsymbol{y}, \tau) \theta(\boldsymbol{y}+\boldsymbol{\eta}, t+\tau)}\right. \\
\left.+\sum_{n=1}^{3} \sum_{m=1}^{3} \pi_{g}^{* n}(\boldsymbol{x}, \boldsymbol{y}, \omega) \pi_{g}^{m}(\boldsymbol{x}, \boldsymbol{y}, \omega) \overline{f_{n}(\boldsymbol{y}, \tau) f_{m}(\boldsymbol{y}+\boldsymbol{\eta}, t+\tau)}\right\} \\
\times \exp \left[\frac{-i \omega}{c_{\infty} x}\left(x_{1} \xi+x_{2} \eta+x_{3} \zeta\right)\right] \exp [-i \omega \tau] d \tau d \boldsymbol{\eta} d \boldsymbol{y} .
\end{array}
$$

Following the source model of Morris and Boluriaan ${ }^{2}$ (which is equivalent to Tam and Auriault ${ }^{3}$ ) for the dilatation rate,

$$
\overline{\theta(\boldsymbol{y}, \tau) \theta(\boldsymbol{y}+\boldsymbol{\eta}, t+\tau)}=A_{s}^{2} \frac{\left(u_{s} / c_{\infty}\right)^{4}}{\tau_{s}^{2}} E(\boldsymbol{\eta}, \tau)
$$

where $A_{s}$ is a constant. The source model of Morris and Boluriaan ${ }^{2}$ for the unsteady force per unit mass is,

$$
\overline{f_{n}(\boldsymbol{y}, \tau) f_{m}(\boldsymbol{y}+\boldsymbol{\eta}, t+\tau)}=B_{s}^{2} \frac{\left(u_{s} / c_{\infty}\right)^{2} u_{s}^{2}}{l_{x}^{2}} E(\boldsymbol{\eta}, \tau)
$$

where $B_{s}$ is a constant. A model must be formed for $E(\boldsymbol{\eta}, \tau)$.

Following Ribner's ${ }^{30}$ postulate, the spatial and temporal terms of the model are separable,

$$
E(\boldsymbol{\eta}, \tau)=\exp \left[\frac{-|\tau|}{\tau_{s}}\right] \exp \left[\frac{-(\xi-\bar{u} \tau)^{2}}{l_{x}^{2}}\right] \exp \left[\frac{-(\eta-\bar{v} \tau)^{2}}{l_{y}^{2}}\right] \exp \left[\frac{-(\zeta-\bar{w} \tau)^{2}}{l_{z}^{2}}\right]
$$

The integrations involving $\boldsymbol{\eta}$ and $\tau$ can be performed analytically. These integrals and associated variables are isolated,

$$
\begin{aligned}
\int_{-\infty}^{\infty} \ldots \int_{-\infty}^{\infty} \exp \left[\frac{-|\tau|}{\tau_{s}}\right] \exp \left[\frac{-(\xi-\bar{u} \tau)^{2}}{l_{x}^{2}}\right] \exp \left[\frac{-(\eta-\bar{v} \tau)^{2}}{l_{y}^{2}}\right] \exp \left[\frac{-(\zeta-\bar{w} \tau)^{2}}{l_{z}^{2}}\right] \\
\times \exp \left[\frac{-i \omega}{c_{\infty} x}\left(x_{1} \xi+x_{2} \eta+x_{3} \zeta\right)\right] \exp [-i \omega \tau] d \xi d \eta d \zeta d \tau
\end{aligned}
$$

The integrations with respect to $\xi, \zeta, \eta$, and $\tau$, results in,

$$
\frac{2 \pi^{3 / 2} c_{\infty}^{2} l_{x} l_{y} l_{z} x^{2} \tau_{s}}{c_{\infty}^{2} x^{2}+\left(c_{\infty} x+\bar{u} x_{1}+\bar{v} x_{2}+\bar{w} x_{3}\right)^{2} \tau_{s}^{2} \omega^{2}} \exp \left[-\frac{\left(l_{x}^{2} x_{1}^{2}+l_{y}^{2} x_{2}^{2}+l_{z}^{2} x_{3}^{2}\right) \omega^{2}}{4 c_{\infty}^{2} x^{2}}\right]
$$

The integrations of $\boldsymbol{\eta}$ and $\tau$ require the assumptions $\tau_{s}>0, l_{i}>0, c_{\infty}>0, x>0$, where each of these is real. These are reasonable assumptions physically as the turbulent kinetic energy at the boundary is zero and we restrict our modeling of the jet flow to $x>0$, where $x=0$ and $-D / 2<r<D / 2$ represents the nozzle exit. We can now write the spectral density as, 


$$
\begin{gathered}
S(\boldsymbol{x}, \omega)=\rho_{\infty}^{2} c_{\infty}^{4} \int_{-\infty}^{\infty} \int_{-\infty}^{\infty} \int_{-\infty}^{\infty} \frac{2 \pi^{3 / 2} c_{\infty}^{2} l_{x} l_{y} l_{z} x^{2} \tau_{s}}{c_{\infty}^{2} x^{2}+\left(c_{\infty} x+\bar{u} x_{1}+\bar{v} x_{2}+\bar{w} x_{3}\right)^{2} \tau_{s}^{2} \omega^{2}} \\
\times\left\{\pi_{g}^{* 0}(\boldsymbol{x}, \boldsymbol{y}, \omega) \pi_{g}^{0}(\boldsymbol{x}, \boldsymbol{y}, \omega) A_{s}^{2} \frac{\left(u_{s} / c_{\infty}\right)^{4}}{\tau_{s}^{2}}+\sum_{n=1}^{3} \sum_{m=1}^{3} \pi_{g}^{* n}(\boldsymbol{x}, \boldsymbol{y}, \omega) \pi_{g}^{m}(\boldsymbol{x}, \boldsymbol{y}, \omega) B_{s}^{2} \frac{\left(u_{s} / c_{\infty}\right)^{2} u_{s}^{2}}{l_{x}^{2}}\right\} \\
\times \exp \left[-\frac{\left(l_{x}^{2} x_{1}^{2}+l_{y}^{2} x_{2}^{2}+l_{z}^{2} x_{3}^{2}\right) \omega^{2}}{4 c_{\infty}^{2} x^{2}}\right] d \boldsymbol{y} .
\end{gathered}
$$

\section{Broadband Shock-Associated Noise}

A separate approach has been developed to predict the BBSAN component of the total jet noise. This approach is based on an acoustic analogy that follows a similar methodology to that outlined for the mixing noise. Thus, the two methodologies are compatible and the results can be summed. The BBSAN component of the jet noise is calculated using the acoustic analogy of Morris and Miller ${ }^{4}$ and its development is briefly discussed here.

The equivalent source terms depend on the product of the jet's turbulent velocity fluctuations and the shock cell structure pressure, and a statistical model is used to describe the properties of the jet turbulence. The model of Morris and Miller is,

$$
\begin{aligned}
& S(\boldsymbol{x}, \omega)=\frac{1}{16 \pi \sqrt{\pi} c_{\infty}^{4} \boldsymbol{x}^{2}} \int_{-\infty}^{\infty} \ldots \int_{-\infty}^{\infty} \frac{K l_{\perp}^{2}}{l \tau_{s}} p_{s}(\boldsymbol{y}) \tilde{p}_{s}\left(k_{1}, y_{2}, y_{3}\right) \\
& \times \frac{\omega^{2} \tau_{s}^{2} \exp \left[-l^{2}\left(k_{1}-\omega \cos \theta / c_{\infty}\right)^{2} / 4-\omega^{2} l_{\perp}^{2} \sin ^{2} \theta / 4 c_{\infty}^{2}\right]}{1+\left(1-M_{c} \cos \theta+\bar{u}_{c} k_{1} / \omega\right)^{2} \omega^{2} \tau_{s}^{2}} d k_{1} d \boldsymbol{y}
\end{aligned}
$$

where $M_{c}$ is the local convective Mach number of the turbulence, $\tilde{p}_{s}\left(k_{1}, y_{2}, y_{3}\right)$ is the Fourier transform of the shock pressure $\left(\bar{p}-p_{\infty}\right)$ in the axial direction, $k_{1}$ is the wavenumber component in the axial direction, and $\bar{u}_{c}$ is the convective velocity of the turbulence in the axial direction.

This BBSAN model uses the vector Green's function of the LEE in a quiescent environment for propagation. Realistic propagation effects can be taken into account by using the approach described by Miller and Morris $^{31}$ or Henry et al. ${ }^{32}$ Since the propagation of the BBSAN through the shear layer is not being captured by the choice of the Green's function, an empirical correction suggested by Tam ${ }^{17}$ was used to calculate the scaling of BBSAN with increasing temperature, but could more accurately be taken into account as shown by Miller. ${ }^{33}$

The integral scales of turbulence in Eqns. 22 and 23 are both based on steady RANS solutions. The length scales within the BBSAN model are chosen to be independent of frequency as there is little effect on the predicted BBSAN with frequency dependent length scales. Since BBSAN is caused by large-scale turbulent structures, small-scale turbulence is not relevant to the calculation.

\section{A Partially Comprehensive Model}

The models for spectral density of mixing noise and BBSAN are now combined into a single model equation.

$$
\begin{array}{r}
S(\boldsymbol{x}, \omega)=\rho_{\infty}^{2} c_{\infty}^{4} \int_{-\infty}^{\infty} \ldots \int_{-\infty}^{\infty}\left\{\pi_{g}^{* 0}(\boldsymbol{x}, \boldsymbol{y}, \omega) \pi_{g}^{0}(\boldsymbol{x}, \boldsymbol{y}, \omega) \overline{\theta(\boldsymbol{y}, \tau) \theta(\boldsymbol{y}+\boldsymbol{\eta}, t+\tau)}\right. \\
+\sum_{n=1}^{3} \sum_{m=1}^{3} \pi_{g}^{* n}(\boldsymbol{x}, \boldsymbol{y}, \omega) \pi_{g}^{m}(\boldsymbol{x}, \boldsymbol{y}, \omega) \overline{f_{n}(\boldsymbol{y}, \tau) f_{m}(\boldsymbol{y}+\boldsymbol{\eta}, t+\tau)} \\
\left.+\sum_{n=1}^{3} \sum_{m=1}^{3} \pi_{g}^{* n}(\boldsymbol{x}, \boldsymbol{y}, \omega) \pi_{g}^{m}(\boldsymbol{x}, \boldsymbol{y}, \omega) \overline{f_{n}^{v}(\boldsymbol{y}, \tau) f_{m}^{v}(\boldsymbol{y}+\boldsymbol{\eta}, t+\tau)}\right\} \\
\times \exp \left[\frac{-i \omega}{c_{\infty} x}\left(x_{1} \xi+x_{2} \eta+x_{3} \zeta\right)\right] \exp [-i \omega \tau] d \tau d \boldsymbol{\eta} d \boldsymbol{y} .
\end{array}
$$

where, 


$$
f_{i}^{v}(\boldsymbol{y}, t)=-u_{s j}^{\prime} \frac{\partial u_{t i}^{\prime}}{\partial x_{j}}-u_{t j}^{\prime} \frac{\partial u_{s i}^{\prime}}{\partial x_{j}}
$$

and $u_{s j}^{\prime}$ and $u_{t i}^{\prime}$ are the velocity perturbation due to the shocks and turbulence respectively. Following Morris and Miller, ${ }^{29}$

$$
\overline{f_{n}^{v}(\boldsymbol{y}, \tau) f_{m}^{v}(\boldsymbol{y}+\boldsymbol{\eta}, t+\tau)}=\frac{a_{m n}(\boldsymbol{y}) p_{s}(\boldsymbol{y}) p_{s}(\boldsymbol{y}+\boldsymbol{\eta}) k(\boldsymbol{y}) E(\boldsymbol{\eta}, \tau)}{\bar{\rho}(\boldsymbol{y})^{2} \bar{u}^{2}(\boldsymbol{y}) l_{x}^{2}(\boldsymbol{y})}
$$

Substituting in the source terms from the mixing and BBSAN model,

$$
\begin{aligned}
& S(\boldsymbol{x}, \omega)=\rho_{\infty}^{2} c_{\infty}^{4} \int_{-\infty}^{\infty} \ldots \int_{-\infty}^{\infty}\left\{\pi_{g}^{* 0}(\boldsymbol{x}, \boldsymbol{y}, \omega) \pi_{g}^{0}(\boldsymbol{x}, \boldsymbol{y}, \omega) A_{s}^{2} \frac{\left(u_{s} / c_{\infty}\right)^{4}}{\tau_{s}^{2}}\right. \\
& \left.+\sum_{n=1}^{3} \sum_{m=1}^{3} \pi_{g}^{* n}(\boldsymbol{x}, \boldsymbol{y}, \omega) \pi_{g}^{m}(\boldsymbol{x}, \boldsymbol{y}, \omega)\left(B_{s}^{2} \frac{\left(u_{s} / c_{\infty}\right)^{2} u_{s}^{2}}{l_{x}^{2}}+\frac{a_{m n} k(\boldsymbol{y}) p_{s}(\boldsymbol{y})}{2 \pi \bar{\rho}^{2} \bar{u}^{2} l_{x}^{2}} \int_{-\infty}^{\infty} \tilde{p}_{s}\left(k_{1}, y_{2}, y_{3}\right) \exp \left[-i k_{1} y_{1}\right]\right)\right\} \\
& \times E(\boldsymbol{\eta}, \tau) \exp \left[\frac{-i \omega}{c_{\infty} x}\left(x_{1} \xi+x_{2} \eta+x_{3} \zeta\right)\right] \exp [-i \omega \tau] d \tau d \boldsymbol{\eta} d \boldsymbol{y} .
\end{aligned}
$$

where,

$$
\tilde{p}_{s}\left(k_{1}, y_{2}, y_{3}\right)=\int_{-\infty}^{\infty} p_{s}(\boldsymbol{y}) \exp \left[i k_{1} y_{1}\right] d y_{1}
$$

and the prefactor $(2 \pi)^{-1}$ is retained in the forward transform. Integrals of Eqn. 27 are isolated,

$$
\int_{-\infty}^{\infty} \cdots \int_{-\infty}^{\infty} E(\boldsymbol{\eta}, \tau) \exp \left[\frac{-i \omega}{c_{\infty} x}\left(x_{1} \xi+x_{2} \eta+x_{3} \zeta\right)\right] \exp [-i \omega \tau] d \tau d \boldsymbol{\eta}
$$

The evaluation of expression 29 and the combination of Eqn. 27, after simplification, results in,

$$
\begin{array}{r}
S(\boldsymbol{x}, \omega)=\rho_{\infty}^{2} c_{\infty}^{4} \int_{-\infty}^{\infty} \int_{-\infty}^{\infty} \int_{-\infty}^{\infty} \frac{2 \pi^{3 / 2} c_{\infty}^{2} l_{x} l_{y} l_{z} x^{2} \tau_{s}}{c_{\infty}^{2} x^{2}+\left(\bar{u} x_{1}+\bar{v} x_{2}+\bar{w} x_{3}+c_{\infty} x\right)^{2} \tau_{s}^{2} \omega^{2}} \\
\times\left\{\pi_{g}^{* 0}(\boldsymbol{x}, \boldsymbol{y}, \omega) \pi_{g}^{0}(\boldsymbol{x}, \boldsymbol{y}, \omega) A_{s}^{2} \frac{\left(u_{s} / c_{\infty}\right)^{4}}{\tau_{s}^{2}}\right. \\
+\sum_{n=1}^{3} \sum_{m=1}^{3} \pi_{g}^{* n}(\boldsymbol{x}, \boldsymbol{y}, \omega) \pi_{g}^{m}(\boldsymbol{x}, \boldsymbol{y}, \omega)\left(B_{s}^{2} \frac{\left(u_{s} / c_{\infty}\right)^{2} u_{s}^{2}}{l_{x}^{2}}\right. \\
\left.\left.+\frac{a_{m n} k(\boldsymbol{y}) p_{s}(\boldsymbol{y})}{2 \pi \bar{\rho}^{2} \bar{u}^{2} l_{x}^{2}} \int_{-\infty}^{\infty} \tilde{p}_{s}\left(k_{1}, y_{2}, y_{3}\right) \exp \left[-i k_{1} y_{1}\right]\right)\right\} \\
\times \exp \left[\frac{-\left(l_{x}^{2} x_{1}^{2}+l_{y}^{2} x_{2}^{2}+l_{z}^{2} x_{3}^{2}\right) \omega^{2}}{4 c_{\infty}^{2} x^{2}}\right] d \boldsymbol{y} .
\end{array}
$$

Equation 30 is a partially comprehensive analogy that contains noise sources for all mixing noise components of jet noise and the broadband component of the shock-associated noise. It does not contain a source term for the discrete component of shock-associated noise. It also assumes there is no additional noise source due to the interaction of the mixing noise source with the shock-associated noise source. This would require a model for an equivalent source of the form $\overline{f_{n}(\boldsymbol{y}, t) f_{m}^{v}(\boldsymbol{y}+\boldsymbol{\eta}, t+\tau)}$ or perhaps $\overline{\theta(\boldsymbol{y}, \tau) f_{m}^{v}(\boldsymbol{y}+\boldsymbol{\eta}, t+\tau)}$. At this time it is unknown what models are appropriate for these potential source terms.

\section{Frequency Dependent Integral Scales}

Equations 22, 23, and 30 require the integral scales of turbulence. Jet flows exhibit self similarity and the length scale grows linearly with increasing streamwise distance. An empirical model of the growth of the length scale with streamwise distance might be approximated as, $l_{x}\left(y_{1}\right) \simeq 0.138 y_{1} D$, where $y_{1}$ is the 
streamwise distance from the nozzle exit to the source. Here, the integral scale of turbulence is found from $K$ and $\epsilon$ directly from the steady RANS solution as,

$$
l_{x}(\boldsymbol{y})=c_{l \operatorname{mix}} K(\boldsymbol{y}) / \epsilon(\boldsymbol{y})
$$

which is the simplest approach that retains the correct dimensions.

Morris and Zaman ${ }^{34}$ examined the variation of the length scale with St. They observed that from low to mid frequencies the integral length scale is relatively independent of $S t$ and at high frequencies the integral length scale falls off as the inverse of St. Morris and Boluriaan ${ }^{2}$ incorporated frequency dependent length scales in their model for jet noise and demonstrated improved predictions. Lieb and Goldstein ${ }^{5}$ examined $^{2}$ frequency dependent length scales and showed their effect on jet noise predictions. After examining multiple choices for an appropriate frequency dependent length scale we select,

$$
l_{x}(\boldsymbol{y}, S t)=l_{x}(\boldsymbol{y}) \frac{1-\exp \left[-c_{f} S t\right]}{c_{f} S t}
$$

where $c_{f}=11.25$ is a coefficient that is set to match the observations of Morris and Zaman. ${ }^{34}$ The temporal and velocity scales must also be related to the steady RANS solution. A simple dimensional model that follows Eqn. 31 is selected. The temporal and velocity integral scales of turbulence are,

$$
\tau_{s}(\boldsymbol{y})=c_{\tau \operatorname{mix}} K(\boldsymbol{y}) / \epsilon(\boldsymbol{y})
$$

and,

$$
u_{s}(\boldsymbol{y})=u_{\text {smix }} \sqrt{2 K(\boldsymbol{y}) / 3}
$$

where $c_{\tau \text { mix }}$ and $u_{s \operatorname{mix}}$ are constant coefficients.

The constants shown in Eqns. 30, 31, 33, and 34 are specified by performing a parametric study relative to a single jet condition and observer angle with various sets of experimental data. The coefficients are $A_{s}=0.25, B_{s}=2.51, c_{\tau m i x}=0.06, u_{s m i x}=1.00$, and $c_{l m i x}=0.40$. The cross-stream length scales are $1 / 3$ of the streamwise length scale, which is a typically accepted value. The effect of using a frequency dependent length scale for mixing noise is justified in terms of representing the physics of turbulence in jets and has improved predictions as shown by Lieb and Goldstein. ${ }^{5}$

\section{Steady RANS Solutions}

The steady RANS solutions are found using the FUN3D solver developed at NASA Langley Research Center. For more information on FUN3D see Anderson and Bonhaus ${ }^{35}$ or Nielsen. ${ }^{36}$ The BBSAN model is compatible with other steady RANS solutions and was demonstrated with the Wind-US code (see Nelson ${ }^{37}$ for details on Wind-US).

In this paper multiple axisymmetric nozzles are examined. A single plane in the streamwise and radial direction of the nozzle is constructed that contains the nozzle interior, exterior, and plume region. This domain contains the exact axisymmetric geometry used in the corresponding experiment. The axisymmetric plane is rotated $90 \mathrm{deg}$. in the azimuthal direction about the centerline axis of the jet $(x=0$ and $y=0)$. 25 planes are created in the azimuthal direction. This operation yields a three-dimensional grid consisting of hexahedrons except on the centerline axis that consists of prisms.

Singlestream axisymmetric nozzles of this study are the SMC000, SMC014, SMC016, and SMC018 of NASA Glenn Research Center. They all have exit diameters of $0.0508 \mathrm{~m}$. Figures 3 and 4 show a region of the computational domain of the SMC000 and SMC016 nozzles respectively. The domain extends $100 D$ downstream from the nozzle exit, $50 D$ in the radial direction from the centerline, and $5 D$ upstream from the nozzle inlet. Another nozzle examined is the Boeing Company axisymmetric dual-stream nozzle shown in Fig. 5, that has a primary exit diameter of $0.06223 \mathrm{~m}$ and a secondary exit diameter of $0.152 \mathrm{~m}$. The ranges of the dual-stream nozzle domain are the same as the single-stream domains. Each of these figures are normalized about the exit diameter of the core stream.

Numerical solutions are governed by the steady RANS equations closed by the Menter SST ${ }^{6}$ turbulence model. Roe ${ }^{38}$ flux vector construction is used for spatial discretization and is second order accurate. A Roe ${ }^{39}$ 'modmin' flux limiter is used in cases that fail to converge due to very large fluxes in the initial transient solution. This approach results in a total variational diminishing scheme. An 'inlet' boundary condition 
is set at the nozzle inflow that holds total pressure and total temperature constant. At the nozzle wall an adiabatic no-slip condition is set and the normal flux is zero. Far-field boundaries are set as 'freestream' with $M_{\infty}=0.01$ for stability and convergence. An 'outflow' boundary condition is set at the downstream faces that hold the static pressure equal to the ambient, $p_{\infty}=101325 \mathrm{~Pa}$. Finally, the $x-y$ and $x-z$ planes, that intersect the nozzle centerline axis, are set as symmetric boundaries. These boundary conditions are the same as those used by Miller and Veltin. ${ }^{40}$

The flowfield is initialized as quiescent at the start of the simulation. Initial iterations use a CourantFriedrichs-Lewy (CFL) number of 1.0 and increase to a CFL number of 50.0 after 250 iterations. The total number of iterations required for convergence varies depending on the nozzle and boundary conditions. Typically the total number of iterations required for convergence is approximately 8000 . Figure 6 shows the global residual per iteration for a typical case. The global residual of $\rho$ has been reduced by three orders of magnitude and is the smallest overall reduction. In all simulations the domain is decomposed using parallel graph partitioning and fill-reducing matrix ordering and run on multiple processors. When the solver finishes the solution is inspected visually, and the residuals are examined to determine if the case has converged. The solutions consisting of $\bar{\rho}, \bar{u}, \bar{v}, \bar{w}, \bar{p}, K$, and $\Omega$ are written to file. The validation of the FUN3D steady RANS solutions for single-stream jets was performed and is presented in an accompanying paper of this conference.

Figure 7 shows example steady RANS solutions in the vicinity of the nozzle exit for two cases. The conditions are a) $M_{d}=1.00, M_{j}=1.47$, and $T T R=3.20$ (Total Temperature Ratio) and b) $M_{d}=1.50$, $M_{j}=1.50$, and $T T R=1.00$. Single planes are shown with contours of the streamwise velocity component. Clearly, the nozzle geometries are captured within the simulation. The first case shows that the shock cell structure is captured if the jet is operating off-design and is taken into account both in the steady RANS simulations and the predictions for mixing noise and BBSAN.

\section{Implementation}

Equations 22, 23, and 30 are implemented in a computer program. The program uses structured or unstructured steady RANS solutions and converts the specific dissipation, $\Omega$, to dissipation $\epsilon=0.09 K \Omega$. The steady RANS solution is interpolated using the inverse weighted distance method onto a new structured grid that encompasses the jet plume. Numerical integration is performed using the new structured domain to approximate the integrals of the model equations. Evaluation of the shock-associated source term requires $\tilde{p}_{s}$. Additional details for calculating $\tilde{p}_{s}$ are shown in Morris and Miller. ${ }^{29}$ The source term for BBSAN has a coefficient $a_{m n}$, and term $\pi_{g}^{n}$, that are approximated jointly with the assumption of Proudman. ${ }^{20}$ Integration ranges and grid point density are varied until the solution is grid independent.

$\pi_{g}^{n}$ is governed by Eqns. 7 and 8. Unfortunately, a full numerical solver for $\pi_{g}^{n}$ is not available to the author but numerical solution techniques have been demonstrated by Tam and Auriault. ${ }^{41}$ Here, a simplified approach has been undertaken. The spreading rates of jets are relatively small and a locally parallel flow assumption is made. Equations 7 and 8 are written about a locally parallel base-flow at each streamwise location. They are reduced to a single third-order differential equation cast in an adjoint form. The steady RANS solution is used to provide the spatial derivatives of density, streamwise velocity, and pressure. Numerical solutions of the differential equation yield the Green's function of Lilley's equation and are related to $\pi_{g}^{n}$. Full details of the approach to calculate $\pi_{g}^{n}$ are described by Miller and Morris. ${ }^{42}$ Using a locally parallel mean flow will have some effect on the predictions in the downstream direction of the jet, particularly for noise sources located within the turning point of the flow. These have been quantified by Tam and Auriault. ${ }^{41}$ The parallel flow assumption does not affect the model development presented in the previous section.

\section{Results}

The developed model is assessed over a wide range of jet Mach numbers and temperature ratios. Singlestream jet cases are shown in Table 1. Each row represents a case with associated design Mach number, fully expanded Mach number, and total temperature ratio. The exit diameter of the single-stream cases (SHJAR database) is $0.0508 \mathrm{~m}$. Dual-stream jet cases are shown in Table 2. A subscript $p$ represents a condition associated with the core stream and a subscript $s$ represents a condition associated with the bypass stream.

The first set of predictions are shown in Figs. 8 through 10 and represent jet flows from a convergent nozzle with $M_{j} \geq 1$. The nozzle geometry is the SMC000 convergent nozzle of Bridges and Brown. ${ }^{43}$ The observers 
are located at six angles from the nozzle exit at a distance of $R / D=100$. Measured data from Bridges and Brown are shown in red. Predictions of mixing, BBSAN, and total noise are shown as blue, green, and black lines respectively. Generally, in the upstream and sideline direction the predicted BBSAN dominates the mixing noise from mid to high frequencies and is dominated by mixing noise in the downstream direction. This is consistent with experimental observation and the breakdown of jet noise spectra by Viswanathan. ${ }^{44}$

Figure 8 shows spectra from a $M_{j}=1.47$ and $T T R=1.00$ jet. The measured data clearly shows BBSAN and screech. At the sideline and upstream directions $(\psi=50$ through 90 deg.) the mixing noise under-predicts the low frequencies in the range of $S t=0.02$ through 0.2 . In the rest of the frequency range BBSAN is dominant. The peak BBSAN frequency is fairly accurately predicted by the model at all angles, and the amplitude is in particularly good agreement, even with strong screech tones in the sideline and upstream directions. At higher frequencies ( $S t=8$ and above) the BBSAN model often under-predicts the measurement by 2 to $4 \mathrm{~dB}$. In the peak noise direction, $\psi=130$ deg., or near the jet axis, $\psi=150$ deg., the mixing noise is about equal to or dominates the BBSAN respectively.

A second case is shown in Fig. 9 with $M_{j}=1.00$ and $T T R=2.70$, that represents a sonic heated jet. Since the jet is sonic it can not support shock waves and the BBSAN source in the model equation is zero. The measured spectra do not show any shock-associated noise component. The predicted mixing noise magnitudes agree within $4 \mathrm{~dB}$ of the measurement at all angles except $\psi=130 \mathrm{deg}$. where the prediction is approximately $6 \mathrm{~dB}$ below measurement. At the sideline direction the mixing noise prediction shape is similar to measurement but over-predicts the peak frequency by $S t \simeq 0.25$. This is not the case in the upstream direction at $\psi=50 \mathrm{deg}$. where the peak frequencies agree. Near the jet downstream axis the effect of the 'large-scale' source is well captured in magnitude. At very high frequencies in this direction the model under-predicts the measurement by $4 \mathrm{~dB}$ near $S t=10$.

Figure 10 shows the spectra for a $M_{j}=1.24$ and $T T R=3.20$ jet. This flow is weakly off-design relative to that shown in Fig. 8 (observe the relative strengths of the BBSAN) and has additional heating. The relative magnitudes of the mixing noise compared to the measurement show similar trends to the previous case even with the addition of heating and a slight increase in $M_{j}$. The frequency peaks of BBSAN are aligned with measurement but under-predict the magnitude, especially in the downstream direction. At some locations such as the sideline, it is illustrative to observe that the total noise is a combination of both mixing and BBSAN, especially so near $S t \simeq 1.0$.

The predictions shown in Figs. 8 through 10 are representative of the first nine cases shown in Table 1. Overall, the amplification of mixing noise due to the propagation through the jet shear layer is captured. This is the case even with the locally parallel mean flow assumption.

We now examine subsonic jet predictions from the convergent SMC000 nozzle. These are shown in Figs. 11 through 13. In all these cases the source of BBSAN is zero. Figure 11 shows a high speed heated subsonic jet operating at $M_{j}=0.90$ and $T T R=3.20$. The predicted peak magnitudes are within $2 \mathrm{~dB}$ of the measurement except for $\psi=130 \mathrm{deg}$. where the prediction is $7 \mathrm{~dB}$ lower than measurement, but the evolution of the spectral shapes from the upstream through downstream direction show a positive trend with respect to measurement. As an example, the predictions at $\psi=50$ relative to $\psi=150$ deg., have a much sharper high magnitude peak. There is also noticeable error in the prediction of the peak frequency that varies between -0.10 and 0.3 St.

Figure 12 shows a prediction for a $M_{j}=0.70$ and $T T R=1.00$ jet. This case, which is an unheated flow, shows similar trends relative to the heated $M_{j}=0.90$ case, implying that the temperature scaling of the model is reasonable at subsonic speeds. Some differences can be seen relative to Fig. 11. For example, at $\psi=150 \mathrm{deg}$. the lower speed case under-predicts the peak jet noise by $4 \mathrm{~dB}$ but predicts the peak magnitude accurately at $\psi=130 \mathrm{deg}$. Unfortunately, the peak frequencies show some of the same shifting as seen in the supersonic cases. The absolute magnitude of the predictions relative to measurement at the peak of the spectra differ by $\pm 3.5 \mathrm{~dB}$.

A final subsonic prediction is shown in Fig. 13 for a $M_{j}=0.50$ and $T T R=1.00$ jet. Similar trends are seen relative to the other subsonic cases. The varying Mach numbers and temperature ratios of the subsonic jets shown are illustrative of cases 'SMC00010' through 'SMC00017' of Table 1.

A series of jets from single-stream convergent-divergent nozzles are now examined. Figure 14 shows the spectra from a $M_{d}=1.185$ (SMC014 nozzle) on-design jet operating at $T T R=1.80$. This jet, relative to the sonic cases discussed previously or the off-design cases, is only slightly more energetic. The spectra are nearly on-design, and only extremely weak shocks are observed in the upstream and sideline direction. In these directions the prediction agrees very well with measurement from low to mid frequencies but under- 
predicts the noise at higher frequencies. This is partly due to the shock noise component that dominates the noise from $S t=0.8$ through 10 at the upstream observer angles. At $\psi=130$ and $\psi=150$ degs. the predicted peak frequency agrees with measurement but the fall-off of the prediction is too large relative to measurement.

We now turn our attention to a family of jets based on the $M_{d}=1.50$ SMC016 nozzle. The predictions relative to measurement are shown in Figs. 15 through 18 representing an unheated jet flow $(T T R=1.00)$ from the subsonic through under-expanded Mach numbers. Figure 15 represents $M_{j}=0.784$ and the spectra should be shockless. Unfortunately, some of the measured data appear to contain very weak shocks even though the plume is shock free.

An over-expanded $M_{j}=1.294$ jet is shown in Fig. 16 and strong BBSAN and screech tones appear. The mixing noise predictions at the upstream and sideline directions agree very well with experiment and the BBSAN predicts the correct peak frequencies. The BBSAN prediction at the sideline and upstream directions, unfortunately, over-predicts the noise near $S t=0.4$; this causes the total noise prediction to be higher than measurement. The prediction at $\psi=130 \mathrm{deg}$. correctly identifies the frequency where the mixing noise and BBSAN have equal intensity near $S t=1.2$. At the highest observer angle the total prediction over-predicts the peak frequency by $S t=0.15$. The BBSAN at this angle has captured the extra energy above $S t=1$ but unfortunately is dominated by an incorrect mixing noise prediction.

Figure 17 shows the on-design $M_{j}=1.50$ unheated case from the SMC016 nozzle. The measurement reflects proper nozzle design as only extremely weak BBSAN can be seen in the upstream direction. Relative to the previous case, the predicted peak magnitude and frequencies in the dominant jet noise direction more closely match experiments. Unfortunately, at $\psi=130 \mathrm{deg}$. the high frequencies are under-predicted by up to $10 \mathrm{~dB}$.

A final prediction for the $M_{d}=1.50$ SMC016 nozzle is shown with $M_{j}=1.734$. In this case the BBSAN dominates the spectrum in the upstream and sideline direction and partly in the downstream. The measurement contains strong screech tones and these have been shown (Andre et al. ${ }^{45}$ ) to have strong effects on BBSAN. Except in the upstream direction, the BBSAN peak is not correctly predicted and is often higher by $S t=0.2$ to 0.3 . The mixing noise prediction at low frequencies in the sideline and upstream direction under-predicts the measured mixing noise. It is likely that for this jet there are at least some non-negligible source terms that affect the mixing noise source due to shocks in the flow.

The last single-stream prediction is performed using the $M_{d}=1.80$ SMC018 nozzle shown in Fig. 19. The jet operates at $M_{j}=1.80$, which is the highest jet Mach number presented (the highest evaluated is 1.828), and is moderately heated at $T T R=1.648$. The measured spectra are almost shock free. At $\psi=130$ deg. the peak prediction is $2 \mathrm{~dB}$ lower than experiment, and at $\psi=150 \mathrm{deg}$. the peak prediction is lower than measurement by $4 \mathrm{~dB}$. In the sideline direction the low frequencies are under-predicted. In the dominant noise direction the high frequencies are under-predicted.

A final prediction is shown in Fig. 20 for a dual-stream axisymmetric jet. The core stream operates at $M_{d}=1.281$ and $T T R_{p}=2.87$ and the fan stream operates at $M_{j s}=0.848$ and $T T R_{s}=1.00$. The nozzle exits of the core and fan streams are off-set as shown in Fig. 5, and both core and fan nozzles are convergent. The core diameter, $D_{p}$, is $0.06223 \mathrm{~m}$ and the observers are at $R / D_{p}=100$. The core stream is operating off-design and the fan stream is subsonic. Since the nozzle exits are off-set, multiple nonoverlapping integration regions are used to evaluate the model. The measured data in Fig. 20 are courtesy of Viswanathan. ${ }^{46}$ Both the mixing and BBSAN predictions at high frequencies over-predict the measurement by significant amounts at all angles. At lower frequencies $(S t \simeq 0.05)$ the opposite is true and the predictions are lower than measurement, however the measurement does not reflect typical low-frequency trends of jet noise. In the upstream and sideline directions the mixing noise and BBSAN peaks are within $2 \mathrm{~dB}$ of the peak measurement. The quality of predictions of the second case in Table 2 is similar to the first.

In almost all the predictions shown the noise is lower than measurement at $\psi=130 \mathrm{deg}$. Also, almost all predictions at $\psi=150 \mathrm{deg}$. had peak intensity close in magnitude to that of measurement. The use of a vector Green's function solver with a locally parallel mean flow assumption, has shown in these calculations that predicting the peak noise intensities is possible, even at very high Mach numbers. The directivity and frequencies of the peak intensities in the downstream direction are not always in agreement with measurement. With the use of a fully three-dimensional vector Green's function solver of the LEE it could be possible to correct these errors at all Mach numbers.

The equivalent sources in this work are based on well known models that are related to steady RANS solutions. Unfortunately, the accuracy of the steady RANS modeling relative to scaling of the sources with 
increasing $M_{j}$ and TTR is questionable. This is apparent in the assessment paper by Georgiadis et al. ${ }^{47}$ where various models in the same CFD code make vastly different predictions. An assessment of FUN3D for the jets examined in this paper had trouble capturing all the flow-features that are essential for accurate jet noise prediction for a wide range of conditions. It is hopeful that in the future more accurate steady RANS turbulence models will be created. Equivalent sources have substantial potential for improvement. One possible improvement is anisotropic effects, such as those examined by Khavaran ${ }^{48}$ or Hunter, ${ }^{49}$ that could substantially improve predictions.

Spectra varying with increasing observer angle, $\psi$, transition from being dominated by noise from turbulence that is more universal to that which is dominated by large-scale coherent structures. This transition represents, in the theory of Tam et al. ${ }^{7}$ the transition between the fine- and large-scale similarity spectra. The first two terms of Eqn. 24, in the context of similarity spectra, could be thought of as fine- and large-scale sources respectively. The dilatation, unsteady forces per unit mass, and broadband shock-associated sources share the same two-point cross-correlation of the turbulent velocity fluctuation. In this theory, there is no assumption that the noise source consists of fine- or large-scale similarity spectra and associated source, or even traditional shear- and self-noise terms.

\section{Conclusion}

The Euler equations are rearranged into an acoustic analogy with arguments consisting of the vector Green's function of the LEE, two-point second order cross-correlation of the equivalent sources, and a steady RANS solution. The equivalent sources are modeled for jet mixing noise following the work of Tam and Auriault ${ }^{3}$ and Morris and Boluriaan ${ }^{2}$ and for BBSAN following the work of Morris and Miller. ${ }^{29}$ A statistical model of the two-point cross-correlation of the velocity fluctuations is used to describe the turbulence within the jet plume. This statistical source model is shared between the mixing and BBSAN sources. These sources reside in a single equation for the total jet noise but do not account for the discrete component of shock-associated noise. Propagation effects controlled by the vector Green's function of the LEE are found with a numerical methodology in conjunction with a locally parallel flow assumption. Propagation effects for BBSAN are also controlled by the Green's function of the LEE and are approximated by spherical spreading.

A single model equation is evaluated for the total noise, mixing noise, and BBSAN with $M_{j}$ ranging from 0.50 to 1.828 and TTR ranging from 1.00 to 3.20. The predictions are compared with experiments of jets operating subsonically through supersonically and at unheated and heated temperatures. This includes transonic and off-design cases. Predictions generally capture the scaling of both mixing noise and BBSAN for the conditions examined, but some discrepancies remain that are due to the accuracy of steady RANS turbulence model closures, the equivalent sources, and the use of a simplified vector Green's function solver of the linearized Euler equations. The model makes no assumptions regarding the fine- or large-scale turbulence, self- or shear- noise, or convective amplification. Improved predictions can likely be made with the use of a fully three-dimensional vector Green's function solver and improved source and turbulence models.

\section{Acknowledgments}

This work is inspired by the creative ideas of Professors Philip J. Morris, Feri Farassat, and Christopher K. W. Tam. The single stream experimental data are courtesy of Drs. James Bridges and Cliff Brown of the NASA Glenn Research Center at Lewis Field. Dual-stream experimental data are courtesy of Dr. Krishna Viswanathan of the Boeing Company. The author is grateful for continuous support from the National Aeronautics and Space Administration Fundamental Aeronautics Program High Speed Project. 


\section{References}

${ }^{1}$ Morris, P. J. and Farassat, F., "Acoustic Analogy and Alternative Theories for Jet Noise Prediction," AIAA Journal, Vol. 40, No. 4, 2002, pp. 671-680.

${ }^{2}$ Morris, P. J. and Boluriaan, S., "The Prediction of Jet Noise From CFD Data," 10th AIAA/CEAS Aeroacoustics Conference, AIAA Paper 2004-2977, 2004.

${ }^{3}$ Tam, C. K. W. and Auriault, L., "Jet Mixing Noise from Fine-Scale Turbulence," AIAA Journal, Vol. 37, No. 2, 1999, pp. $145-153$.

${ }^{4}$ Morris, P. J. and Miller, S. A. E., "The Prediction of Broadband Shock-Associated Noise Using RANS CFD," 15th AIAA/CEAS Aeroacoustics Conference (30th AIAA Aeroacoustics Conference), AIAA Paper 2009-3315, 2009.

${ }^{5}$ Leib, S. and Goldstein, M. E., "Hybrid Source Model for Predicting High-Speed Jet Noise," AIAA Journal, Vol. 49, No. 7, 2011, pp. 1324-1335.

${ }^{6}$ Menter, F. R., "Two-Equation Eddy-Viscosity Turbulence Models for Engineering Applications," AIAA Journal, Vol. 32, No. 8, 1994, pp. 1598-1605.

${ }^{7}$ Tam, C. K. W., Golebiowski, M., and Seiner, J. M., "On the Two Components of Turbulent Mixing Noise from Supersonic Jets," AIAA Paper 96-1716, 1996.

${ }^{8}$ Ffowcs Williams, J. E., "Hydrodynamic Noise," Annual Review of Fluid Mechanics, Vol. 1, 1969, pp. $197-222$.

${ }^{9}$ Ffowcs Williams, J. E., "Aeroacoustics," Annual Review of Fluid Mechanics, Vol. 9, 1977, pp. 447-68.

${ }^{10}$ Goldstein, M. E., "Aeroacoustics of Turbulent Shear Flows," Annual Review of Fluid Mechanics, Vol. 16, 1984, pp. 263285.

${ }^{11}$ Tam, C. K. W., "Supersonic Jet Noise," Annual Review of Fluid Mechanics, Vol. 27, 1995, pp. 17-43.

${ }^{12}$ Tam, C. K. W., "Mach Wave Radiation from High-Speed Jets," AIAA Journal, Vol. 47, No. 10, 2009, pp. 2440-2448.

${ }^{13}$ Powell, A., "On the Noise Emanating from a Two-Dimensional Jet above the Critical Pressure," Aeronautical Quarterly, Vol. 4, 1953, pp. 103-122.

${ }^{14}$ Raman, G., "Supersonic Jet Screech: Half-Century from Powell to the Present," Journal of Sound and Vibration, Vol. 222, No. 3, 1999, pp. 543-571.

${ }^{15}$ Harper-Bourne, M. and Fisher, M. J., "The Noise from Shock-Waves in Supersonic Jets," AGARD Conference Proceedings, 1973.

${ }^{16}$ Tam, C. K. W., "Stochastic Model Theory of Broadband Shock-Associated Noise from Supersonic Jets," Journal of Sound and Vibration, Vol. 116, No. 2, 1987, pp. 265-302.

${ }^{17}$ Tam, C. K. W., "Broadband Shock-Associated Noise of Moderately Imperfectly-Expanded Supersonic Jets," Journal of Sound and Vibration, Vol. 140, No. 1, 1990, pp. 55-71.

${ }^{18}$ Lighthill, M. J., "On Sound Generated Aerodynamically. I. General Theory," Proc. R. Soc. Lond. A., Vol. 211, No. 1107, 1952 , pp. 564-587.

${ }^{19}$ Lighthill, M. J., "On Sound Generated Aerodynamically. II. Turbulence as a Source of Sound," Proc. R. Soc. Lond. A., Vol. 222, No. 1148, 1954, pp. 1-32.

${ }^{20}$ Proudman, I., "The Generation of Noise by Isotropic Turbulence," Proc. R. Soc. Lond. A, Vol. 214, No. 1116, 1952, pp. 119-132.

${ }^{21}$ Heisenberg, W., "On the Theory of Statistical and Isotropic Turbulence," Proc. R. Soc. Lond. A, Vol. 195, No. 1042, 1949, pp. 402-406.

${ }^{22}$ Curle, N., "The Influence of Solid Boundaries upon Aerodynamic Sound," Proc. Roy. Soc. A, Vol. 231, No. 1187, 1955, pp. 505-514.

${ }^{23}$ Ffowcs Williams, J. E. and Hawkings, D. L., "Sound Generation by Turbulence and Surfaces in Arbitrary Motion," Philosophical Transactions of the Royal Society of London. Series A, Mathematical and Physical Sciences, Vol. 264, No. 1151, May 1969, pp. 321-342.

${ }^{24}$ Lilley, G. M., "On the Noise from Jets," AGARD Conference Proceedings In Noise Mechanisms, Vol. 13, 1974, pp. 1-11.

${ }^{25}$ Khavaran, A., Krejsa, E. A., and Kim, C. M., "Computation of Supersonic Jet Mixing Noise for an Axisymmetric Convergent-Divergent Nozzle," Journal of Aircraft, Vol. 31, No. 3, 1994, pp. 603-609.

${ }^{26}$ Mani, R., Stringas, E. J., Wang, J. C. F., Balsa, T. F., Gliebe, P. R., and Kantola, R. A., "High Velocity Jet Noise Source Location and Reduction," FAA-RD-76-79-II, 1977.

${ }^{27}$ Reichardt, H., "On a New Theory of Free Turbulence," Royal Aeronautical Society Journal, Vol. 47, 1943, pp. 167-176.

${ }^{28}$ Goldstein, M. E., "A Generalized Acoustic Analogy," Journal of Fluid Mechanics, Vol. 488, 2003, pp. 315-333.

${ }^{29}$ Morris, P. J. and Miller, S. A. E., "Prediction of Broadband Shock-Associated Noise Using Reynolds-Averaged NavierStokes Computational Fluid Dynamics," AIAA Journal, Vol. 48, No. 12, 2010, pp. 2931-2961.

${ }^{30}$ Ribner, H. S., "The Generation of Sound by Turbulent Jets," Advances in Applied Mechanics, Vol. 8, 1964, pp. 104-182.

${ }^{31}$ Miller, S. A. E. and Morris, P. J., "The Prediction of Broadband Shock-Associated Noise Including Propagation Effects," 17th AIAA/CEAS Aeroacoustics Conference (32nd AIAA Aeroacoustics Conference), AIAA Paper 2011-2923, 2011.

${ }^{32}$ Henry, C., Bailly, C., and Bodard, G., "Statistical Modeling of BBSAN Including Refraction Effects," 18th AIAA/CEAS Aeroacoustics Conference (33rd AIAA Aeroacoustics Conference), AIAA Paper 2012-2163, 2012.

${ }^{33}$ Miller, S. A. E., "The Scaling of Broadband Shock-Associated Noise with Increasing Temperature," 18th AIAA/CEAS Aeroacoustics Conference (33rd AIAA Aeroacoustics Conference), Colorado Springs, Colorado, AIAA Paper 2012-2215, 2012.

${ }^{34}$ Morris, P. J. and Zaman, K. B. M. Q., "Velocity Measurements in Jets with Application to Noise Source Modeling," Journal of Sound and Vibration, Vol. 329, No. 4, 2010, pp. 394-414.

${ }^{35}$ Anderson, W. K. and Bonhaus, D. L., "An Implicit Upwind Algorithm for Computing Turbulent Flows on Unstructured Grids," Computers and Fluids, Vol. 23, No. 1, 1994, pp. 1-22. 
${ }^{36}$ Nielsen, E. J., Aerodynamic Design Sensitivities on an Unstructured Mesh using the Navier-Stokes Equations and a Discrete Adjoint Formulation, Ph.D. thesis, Virginia Polytechnic Institute and State University, 1998.

${ }^{37}$ Nelson, C., "An Overview of the NPARC Alliance's Wind-US Flow Solver," AIAA Paper 2010-27, 2010.

${ }^{38}$ Roe, P. L., "Approximate Riemann Solvers, Parameter Vectors, and Difference Schemes," Journal of Computational Physics, Vol. 135, No. 2, 1981, pp. 357-372.

${ }^{39}$ Roe, P. L., "Characteristic-based schemes for the Euler Equations," Ann. Rev. Fluid Mech., Vol. 18, 1986, pp. 337-365.

${ }^{40}$ Miller, S. A. E. and Veltin, J., "Experimental and Numerical Investigation of Flow Properties of Supersonic Helium-Air Jets," AIAA Journal, Vol. 29, No. 1, 2011, pp. 235-246.

${ }^{41}$ Tam, C. K. W. and Auriault, L., "Mean Flow Refraction Effects on Sound Radiated from Localized Sources in a Jet," Journal of Fluid Mechanics, Vol. 370, 1998, pp. 149-174.

${ }^{42}$ Miller, S. A. E. and Morris, P. J., "The Prediction of Broadband Shock-Associated Noise Including Propagation Effects," International Journal of Aeroacoustics, Vol. 11, No. 8, 2012, pp. 755-782.

${ }^{43}$ Bridges, J. and Brown, C. A., "Validation of the Small Hot Jet Acoustic Rig for Aeroacoustic Research," 11th AIAA/CEAS Aeroacoustics Conference, Monterey, California, 23 - 25 May, AIAA Paper 2005-2846, 2005.

${ }^{44}$ Viswanathan, K., "Scaling Laws and a Method for Identifying Components of Jet Noise," AIAA Journal, Vol. 44, No. 10, 2006, pp. 2274-2285.

${ }^{45}$ Andre, B., Castelain, T., and Bailly, C., "Broadband Shock-Associated Noise in Screeching and Non-Screeching Underexpanded Supersonic Jets," AIAA Journal, Vol. 51, No. 3, 2013, pp. 665-673.

${ }^{46}$ Viswanathan, K., "Parametric study of noise from dual-stream nozzles," Journal of Fluid Mechanics, Vol. 521, December 2004, pp. 35-68.

${ }^{47}$ Georgiadis, N. J., Yoder, D. A., and Engblom, W. A., "Evaluation of Modified Two-Equation Turbulence Models for Jet Flow Predictions," AIAA Aerospace Sciences Meeting and Exhibit AIAA 2006-490, 2006.

${ }^{48}$ Khavaran, A., "Role of Anisotropy in Turbulent Mixing Noise," AIAA Journal, Vol. 37, No. 7, 1999, pp. 832-841.

${ }^{49}$ Hunter, C. A., An Approximate Jet Noise Prediction Method based on Reynolds-Averaged Navier-Stokes Computational Fluid Dynamics, Ph.D. thesis, The George Washington University, 2002.

${ }^{50}$ Zaman, K. B. M. Q., Bencic, T., Clem, M., and Fagan, A., "Shock-Induced Boundary Layer Separation in C-D Nozzles and Its Impact on Jet Noise," 49th AIAA Aerospace Sciences Meeting, Orlando, Florida, AIAA Paper 2011-1031,2011. 
Table 1. Single-stream jet operating conditions. Case names with a ${ }^{*}$, are shown in this paper.

\begin{tabular}{|c|c|c|c|}
\hline Case & $M_{d}$ & $M_{j}$ & $T T R$ \\
\hline SMC0001 & 1.00 & 1.00 & 1.00 \\
\hline SMC0002 & 1.00 & 1.24 & 1.00 \\
\hline SMC0003* & 1.00 & 1.47 & 1.00 \\
\hline SMC0004* & 1.00 & 1.00 & 2.70 \\
\hline SMC0005 & 1.00 & 1.24 & 2.70 \\
\hline SMC0006 & 1.00 & 1.47 & 2.70 \\
\hline SMC0007 & 1.00 & 1.00 & 3.20 \\
\hline SMC0008* & 1.00 & 1.24 & 3.20 \\
\hline SMC0009 & 1.00 & 1.47 & 3.20 \\
\hline SMC00010 & 1.00 & 0.70 & 3.20 \\
\hline SMC00011* & 1.00 & 0.90 & 3.20 \\
\hline $\mathrm{SMC} 00012^{*}$ & 1.00 & 0.70 & 1.00 \\
\hline SMC00013 & 1.00 & 0.90 & 1.00 \\
\hline SMC00014* & 1.00 & 0.50 & 1.00 \\
\hline SMC00015 & 1.00 & 0.50 & 1.80 \\
\hline SMC00016 & 1.00 & 0.50 & 2.20 \\
\hline SMC00017 & 1.00 & 0.50 & 2.70 \\
\hline SMC0141 & 1.185 & 1.185 & 1.00 \\
\hline $\mathrm{SMC} 0142^{*}$ & 1.185 & 1.185 & 1.80 \\
\hline SMC0143 & 1.185 & 1.185 & 3.00 \\
\hline SMC0161* & 1.50 & 0.784 & 1.00 \\
\hline SMC0162 & 1.50 & 1.046 & 1.00 \\
\hline SMC0163* & 1.50 & 1.294 & 1.00 \\
\hline SMC0164* & 1.50 & 1.500 & 1.00 \\
\hline SMC0165* & 1.50 & 1.734 & 1.00 \\
\hline SMC0166 & 1.50 & 1.828 & 1.00 \\
\hline SMC0181 & 1.80 & 1.80 & 1.00 \\
\hline SMC0182* & 1.80 & 1.80 & 1.648 \\
\hline
\end{tabular}

Table 2. Dual-stream jet operating conditions. Case names with a ${ }^{* *}$, are shown in this paper.

\begin{tabular}{cccccccc} 
Case & $D_{p}(\mathrm{~m})$ & $D_{s}(\mathrm{~m})$ & $M_{d p}$ & $M_{j p}$ & $M_{j s}$ & $T T R_{p}$ & $T T R_{s}$ \\
\hline axi5* $^{*}$ & 0.06223 & 0.152 & 1.00 & 1.281 & 0.848 & 2.87 & 1.00 \\
axi7 & 0.06223 & 0.152 & 1.00 & 1.240 & 1.358 & 2.87 & 1.00
\end{tabular}

16 of 27 

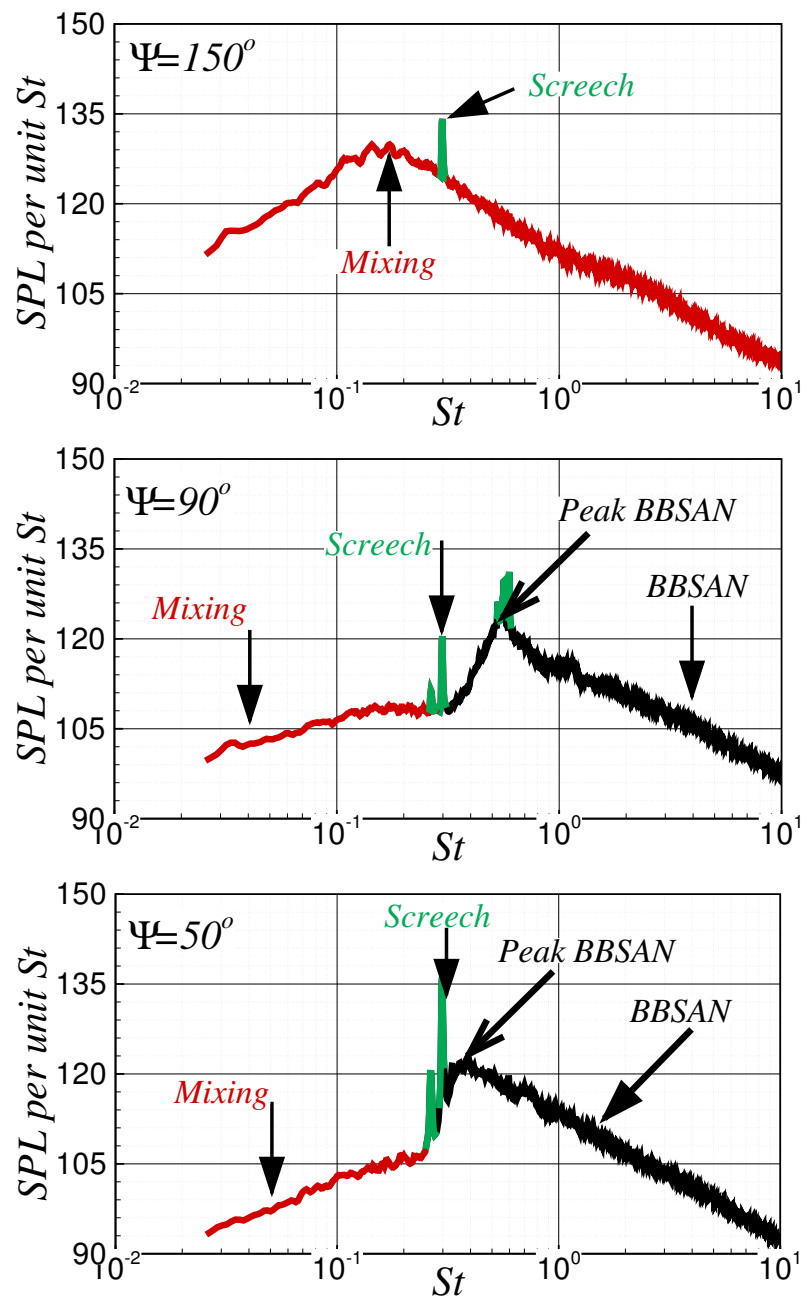

Figure 1. Sound pressure level per unit Strouhal number resulting from a $M_{d}=1.00, M_{j}=1.50$, and $T T R=1.00$ jet. The red lines represent the noise that is dominated by turbulent mixing, black lines represent noise that is dominated by BBSAN, and green lines represent noise that is dominated by discrete tones ('screech'). $\psi$ is the angle from the upstream jet axis to the observer centered about the nozzle exit at $R / D=100$. 


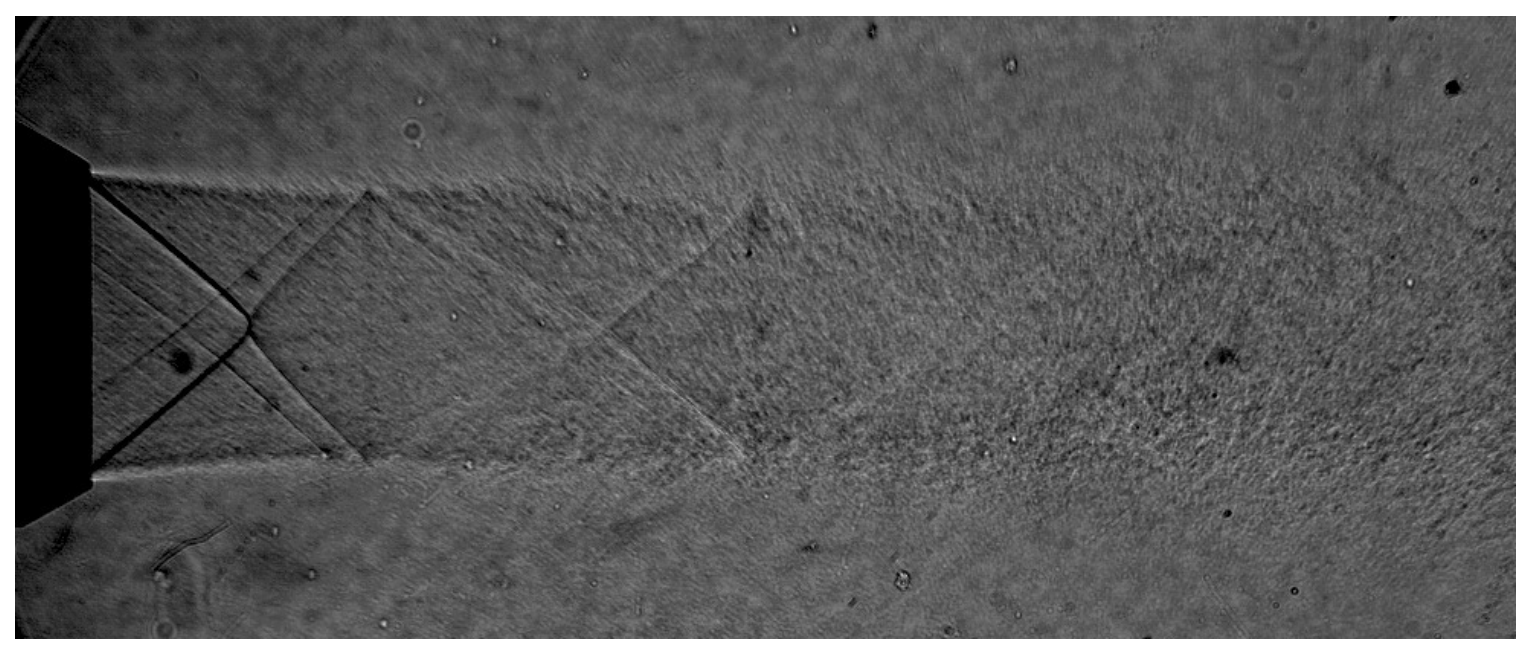

Figure 2. A schlieren of an over-expanded $M_{j}=1.60$ jet from NASA Glenn Research Center courtesy of Zaman et al. ${ }^{50}$ The oblique shock waves and expansion waves are apparent within the jet plume.

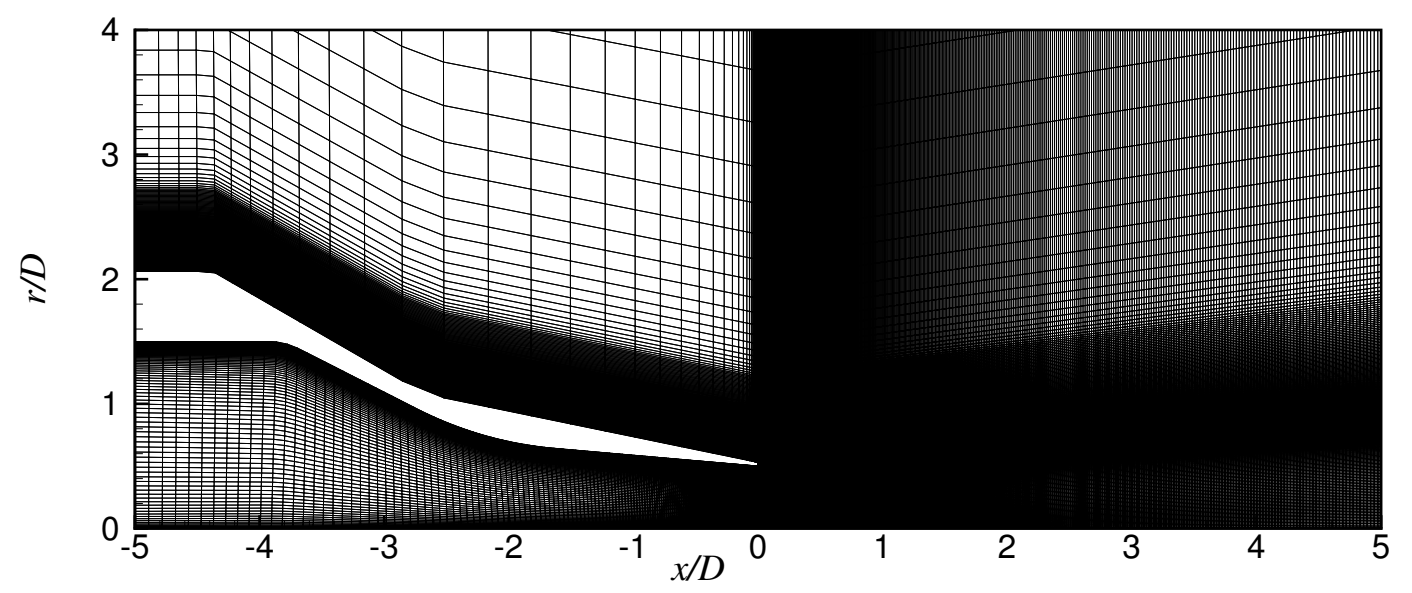

Figure 3. The SMC000 nozzle geometry and a region of the associated computational domain normalized by nozzle diameter. 


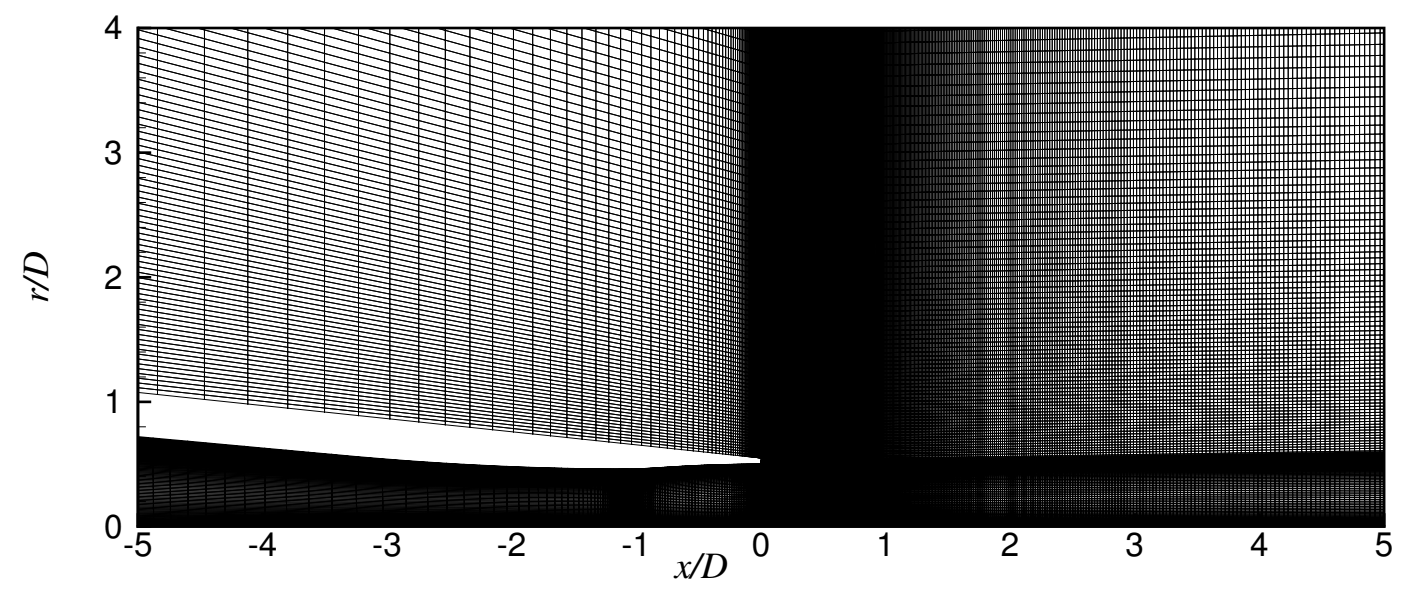

Figure 4. The SMC016 nozzle geometry and a region of the associated computational domain normalized by nozzle diameter.

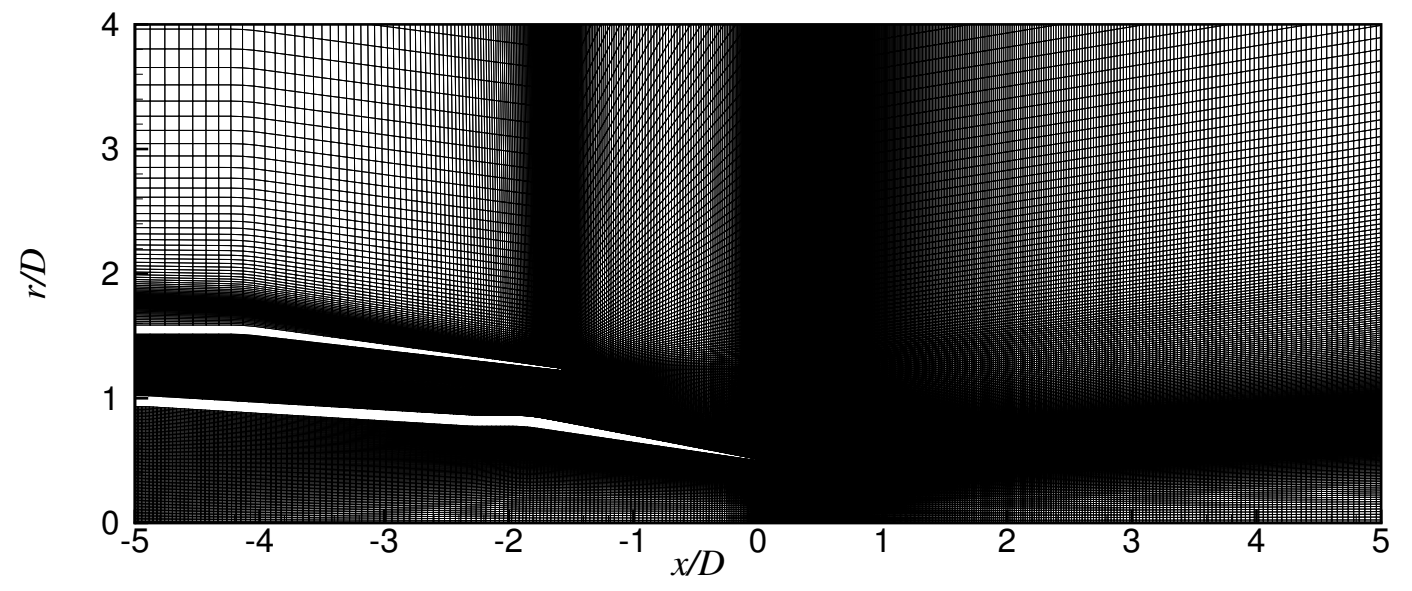

Figure 5. The Boeing dual-stream nozzle geometry and a region of the associated computational domain normalized by nozzle primary diameter. 


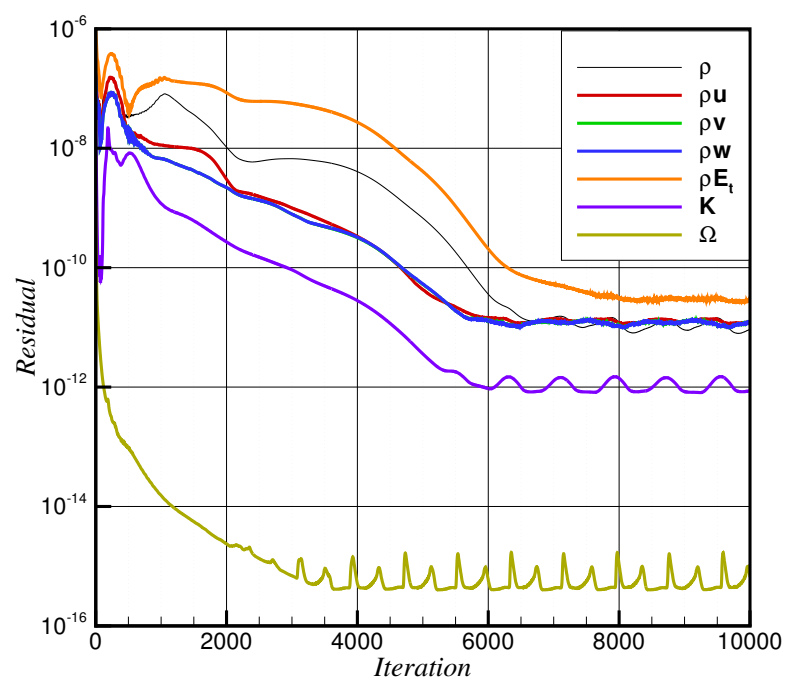

Figure 6. Global residuals calculated by FUN3D of the field variables per iteration for the $M_{d}=1.00, M_{j}=1.47$, $T T R=3.20$ jet flow.
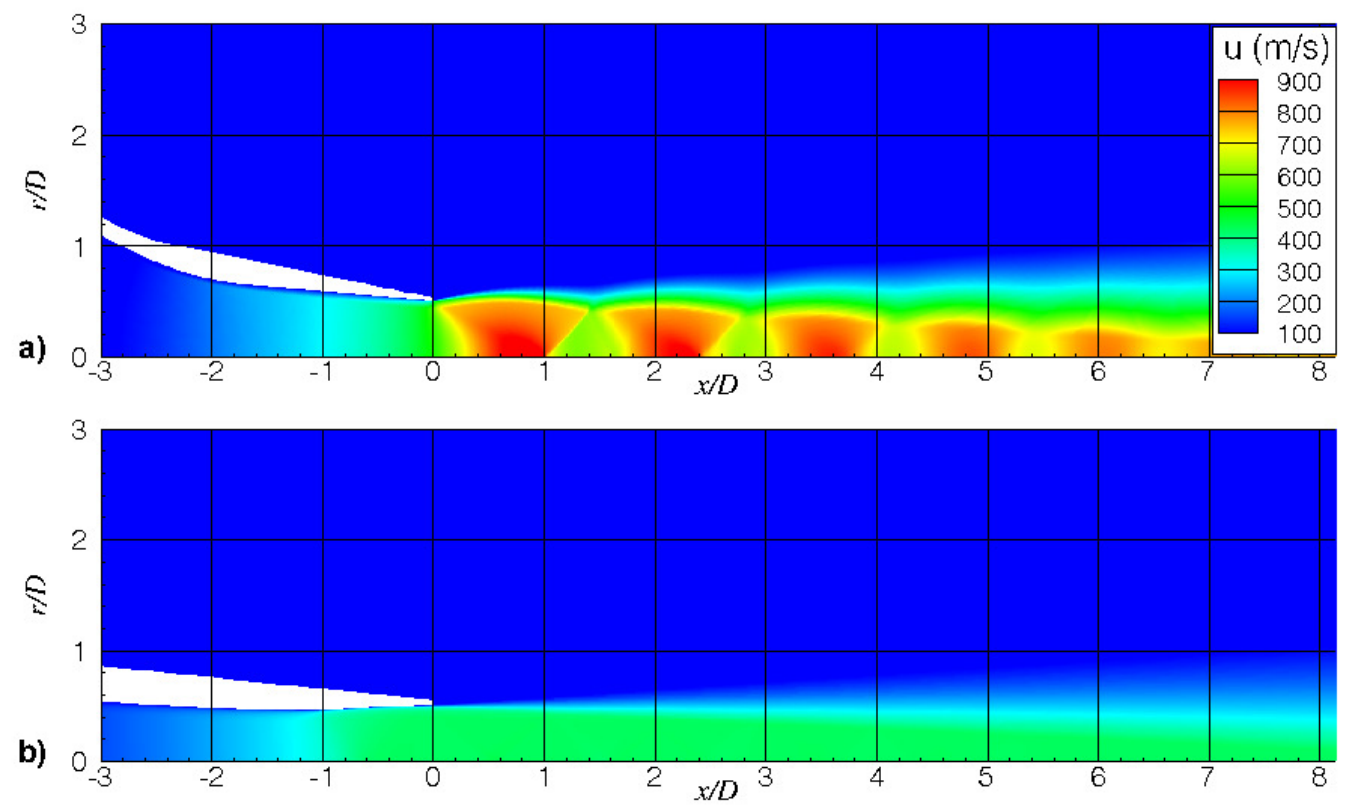

Figure 7. Example steady RANS solutions of the streamwise velocity component of a) $M_{d}=1.00, M_{j}=1.47$, and $T T R=3.20$ and b) $M_{d}=1.50, M_{j}=1.50$, and $T T R=1.00$. 

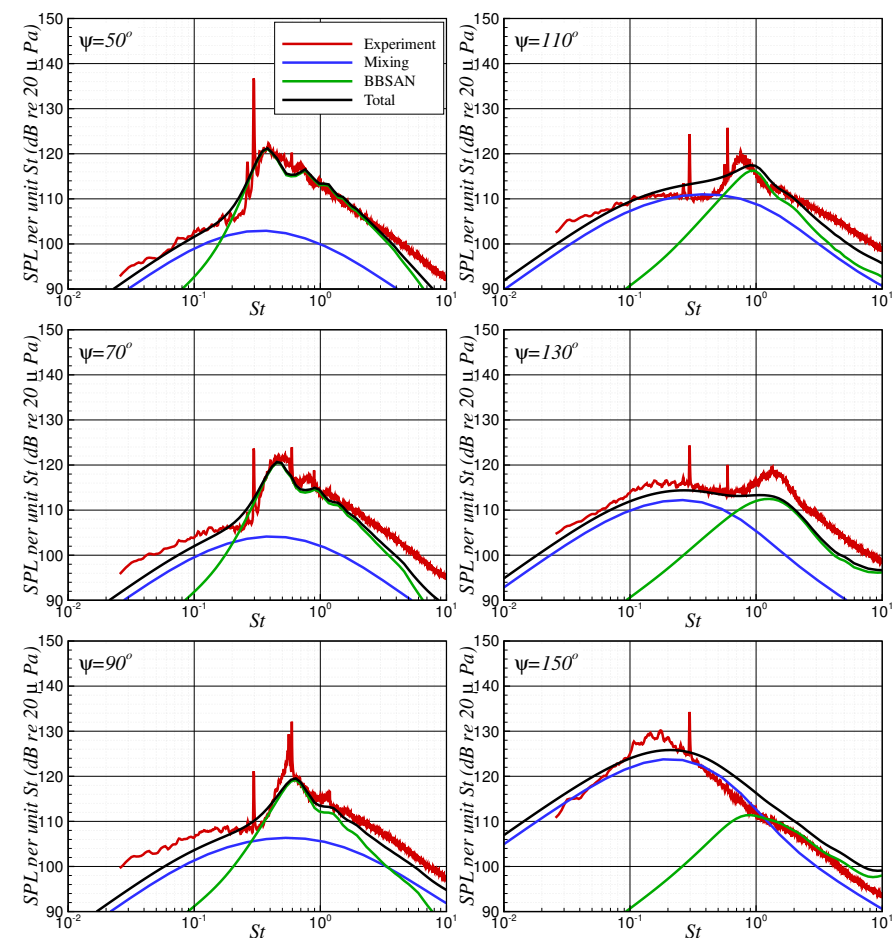

Figure 8. Comparison of predictions (Case SMC0003) with measurement of the SMC000 convergent nozzle operating at $M_{j}=1.47$ and $T T R=1.00$ at a distance of $R / D=100$ and angles $\psi$. 

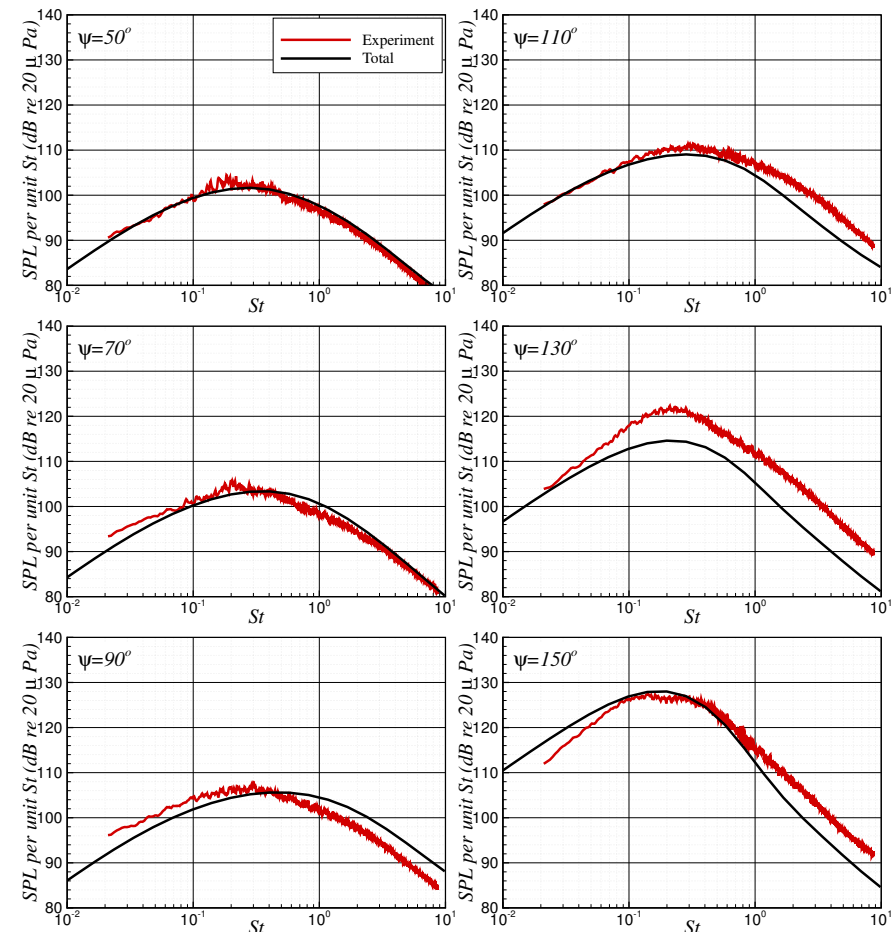

Figure 9. Comparison of predictions (Case SMC0004) with measurement of the SMC000 convergent nozzle operating at $M_{j}=1.00$ and $T T R=2.70$ at a distance of $R / D=100$ and angles $\psi$.
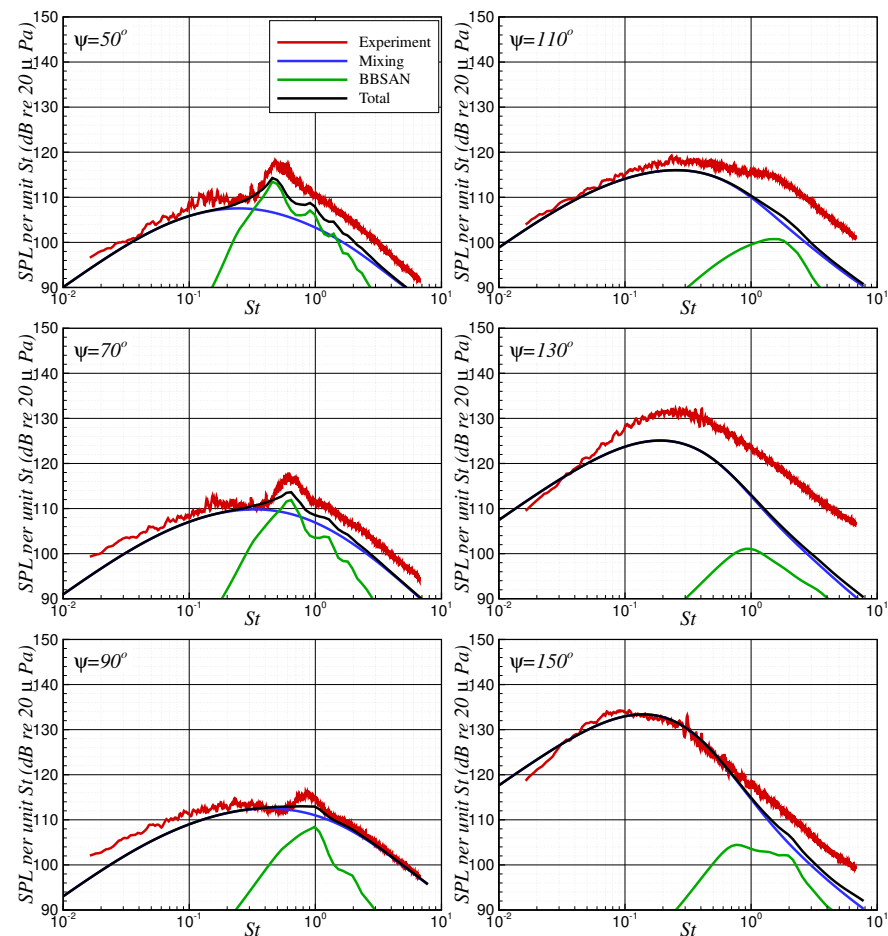

Figure 10. Comparison of predictions (Case SMC0008) with measurement of the SMC000 convergent nozzle operating at $M_{j}=1.24$ and $T T R=3.20$ at a distance of $R / D=100$ and angles $\psi$. 

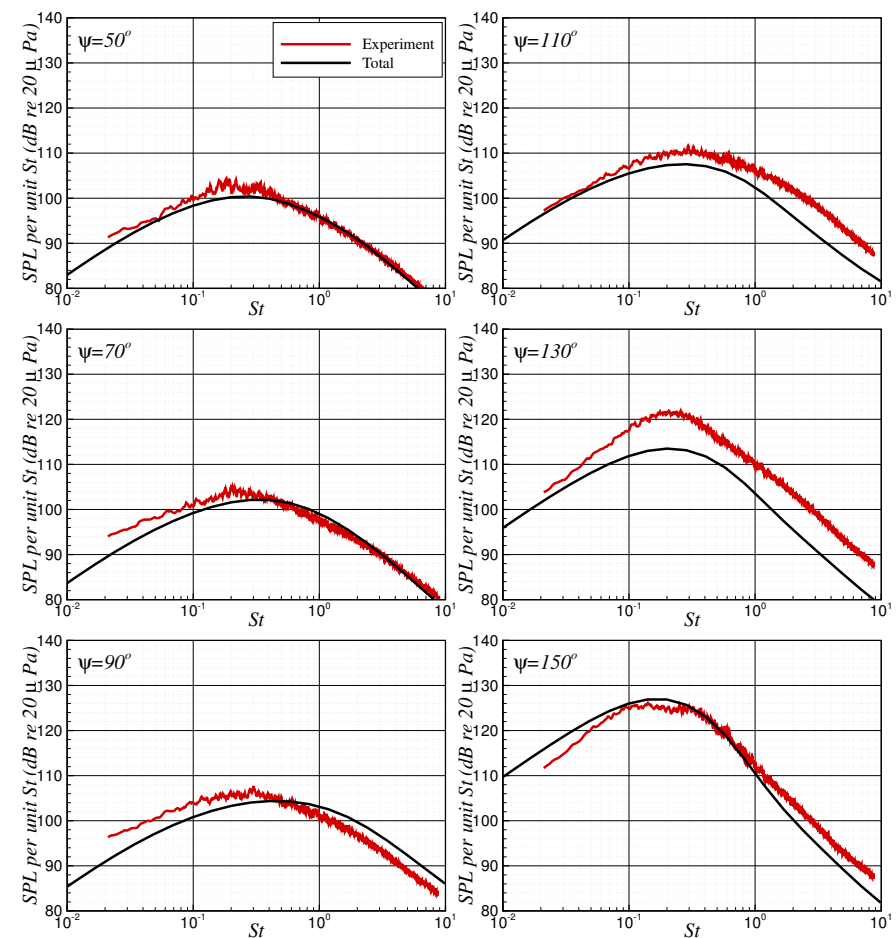

Figure 11. Comparison of predictions (Case SMC00011) with measurement of the SMC000 convergent nozzle operating at $M_{j}=0.90$ and $T T R=3.20$ at a distance of $R / D=100$ and angles $\psi$.
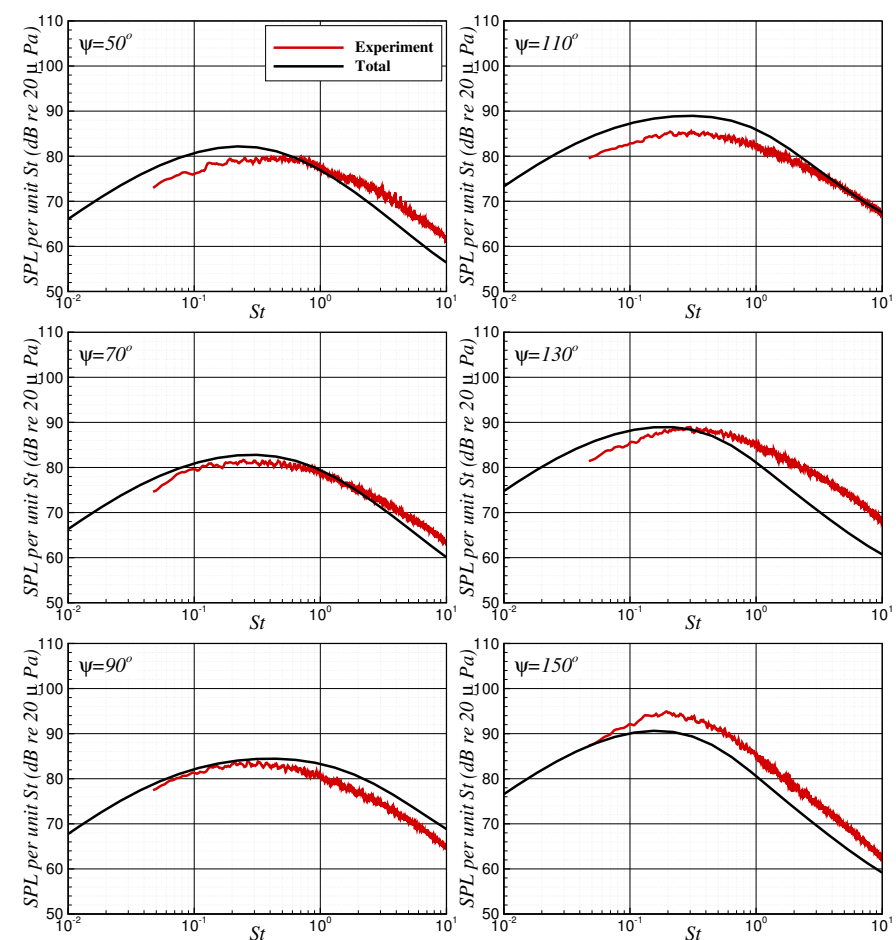

Figure 12. Comparison of predictions (Case SMC00012) with measurement of the SMC000 convergent nozzle operating at $M_{j}=0.70$ and $T T R=1.00$ at a distance of $R / D=100$ and angles $\psi$. 

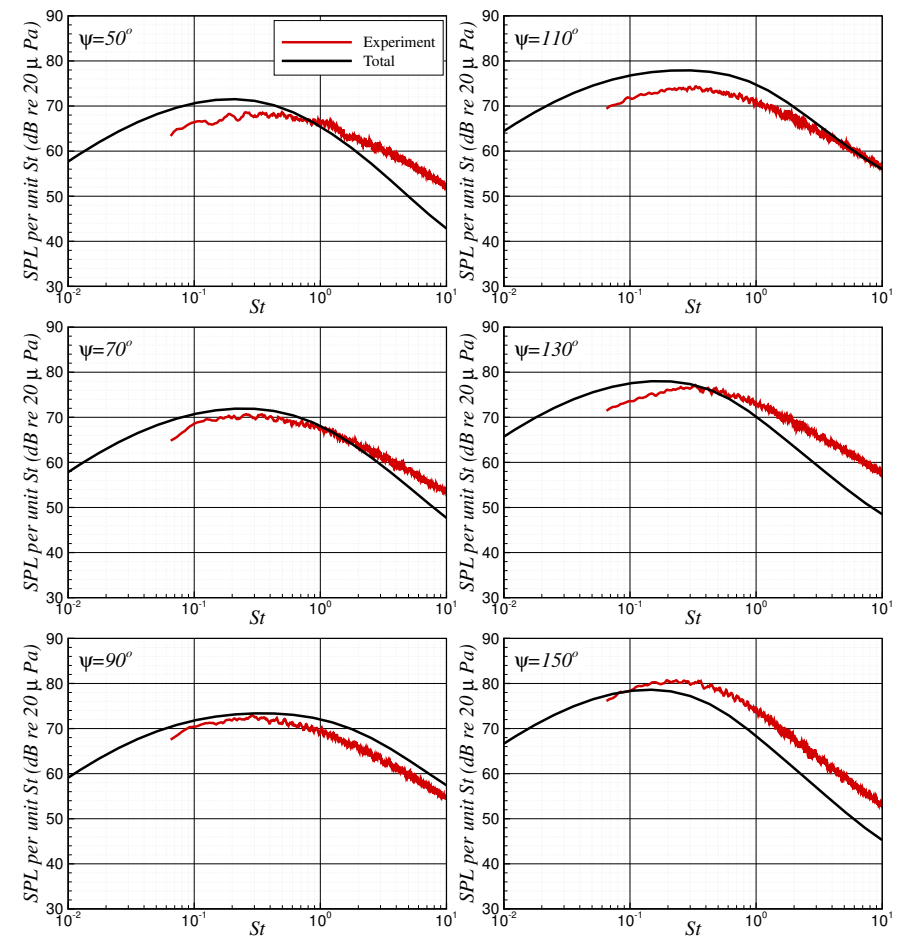

Figure 13. Comparison of predictions (Case SMC00014) with measurement of the SMC000 convergent nozzle operating at $M_{j}=0.50$ and $T T R=1.00$ at a distance of $R / D=100$ and angles $\psi$.
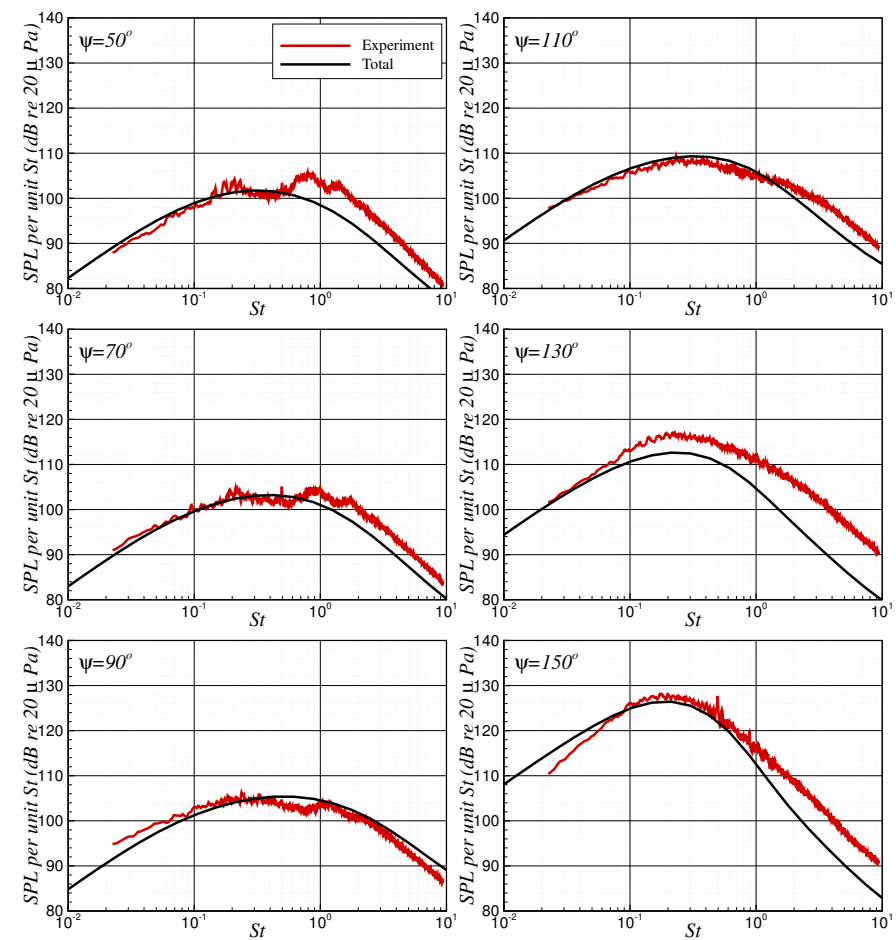

Figure 14. Comparison of predictions (Case SMC0142) with measurement of the SMC014 convergent-divergent nozzle, $M_{d}=1.185$, operating at $M_{j}=1.185$ and $T T R=1.80$ at a distance of $R / D=100$ and angles $\psi$. 

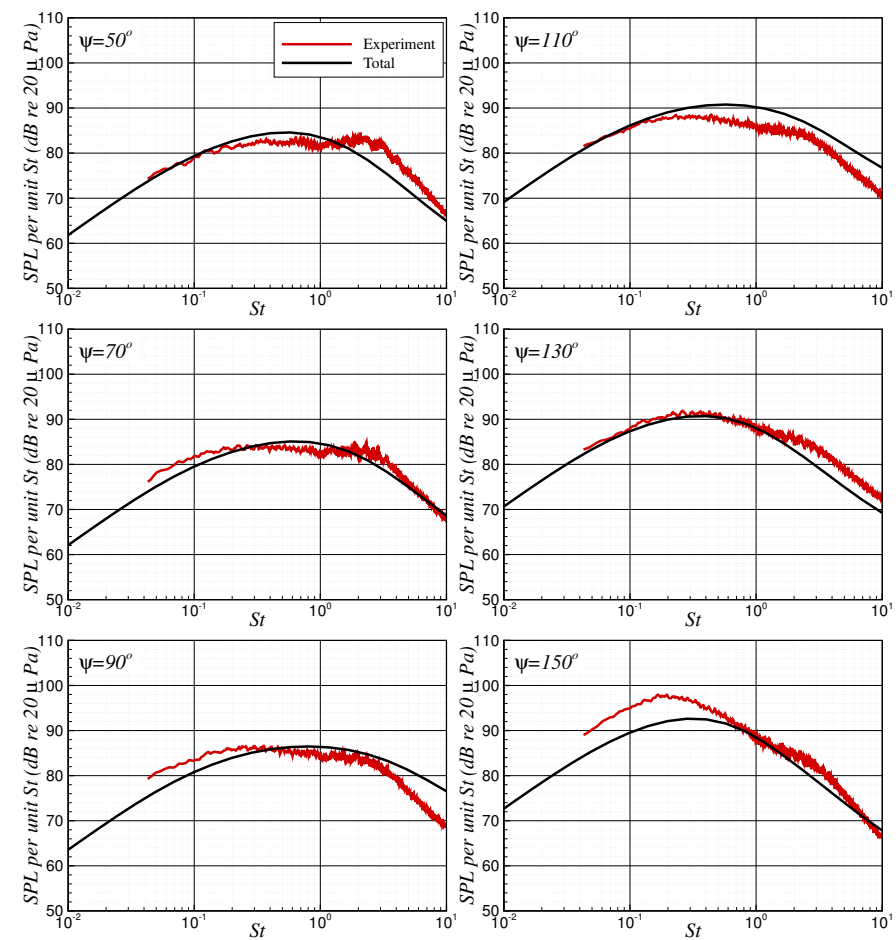

Figure 15. Comparison of predictions (Case SMC0161) with measurement of the SMC016 convergent-divergent nozzle, $M_{d}=1.50$, operating at $M_{j}=0.784$ and $T T R=1.00$ at a distance of $R / D=100$ and angles $\psi$.
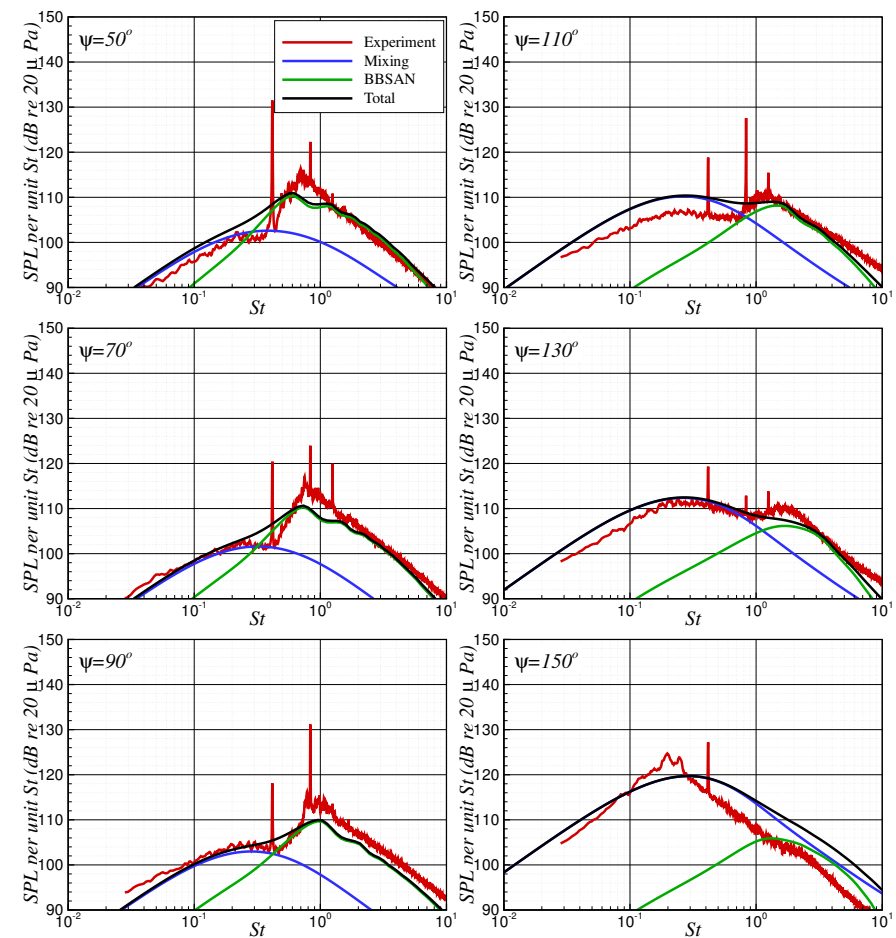

Figure 16. Comparison of predictions (Case SMC0163) with measurement of the SMC016 convergent-divergent nozzle, $M_{d}=1.50$, operating at $M_{j}=1.294$ and $T T R=1.00$ at a distance of $R / D=100$ and angles $\psi$. 

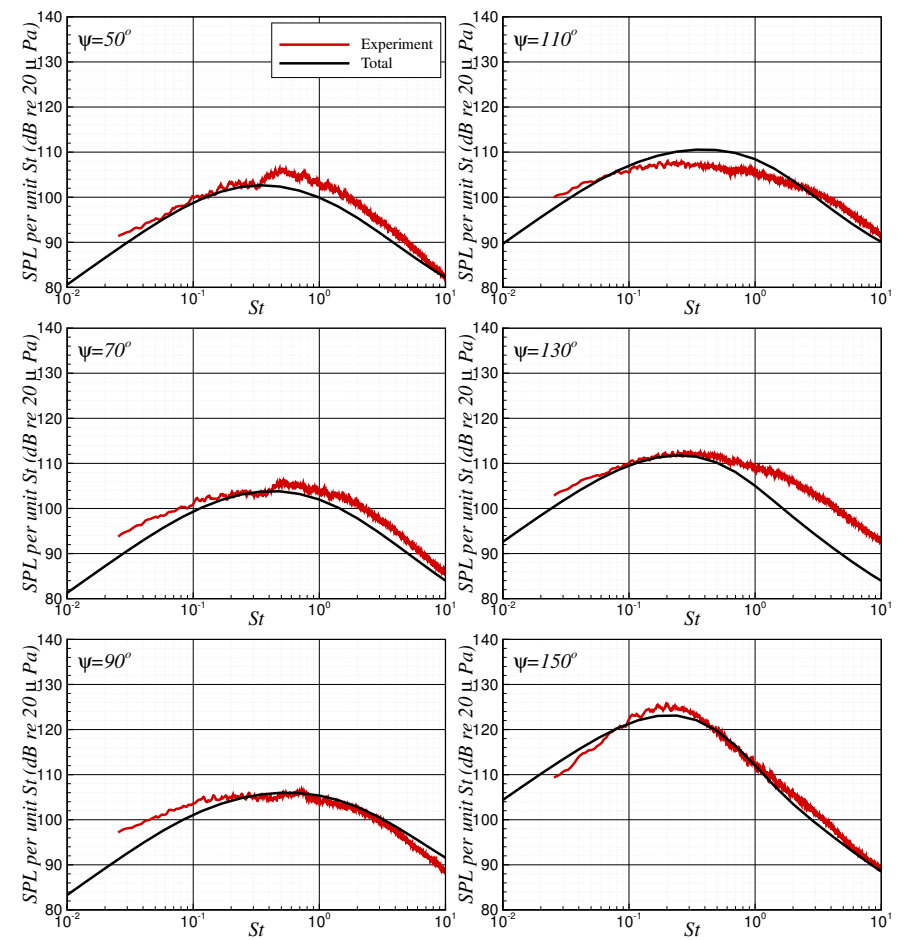

Figure 17. Comparison of predictions (Case SMC0164) with measurement of the SMC016 convergent-divergent nozzle, $M_{d}=1.50$, operating at $M_{j}=1.50$ and $T T R=1.00$ at a distance of $R / D=100$ and angles $\psi$.
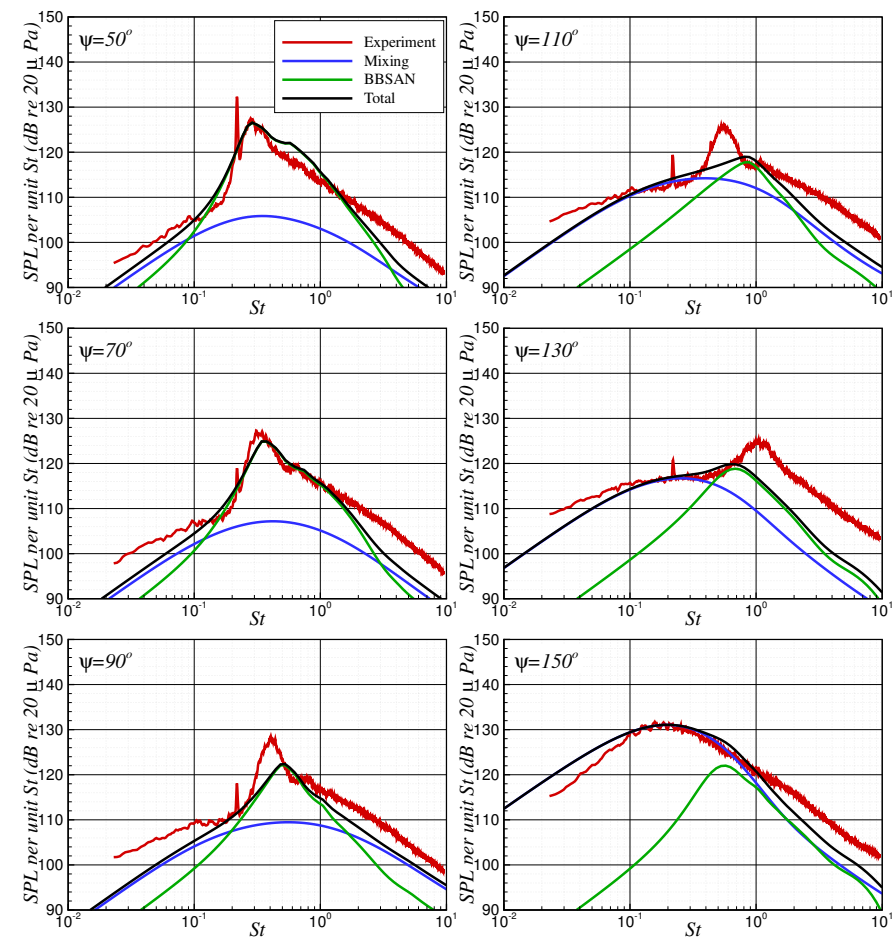

Figure 18. Comparison of predictions (Case SMC0165) with measurement of the SMC016 convergent-divergent nozzle, $M_{d}=1.50$, operating at $M_{j}=1.734$ and $T T R=1.00$ at a distance of $R / D=100$ and angles $\psi$. 

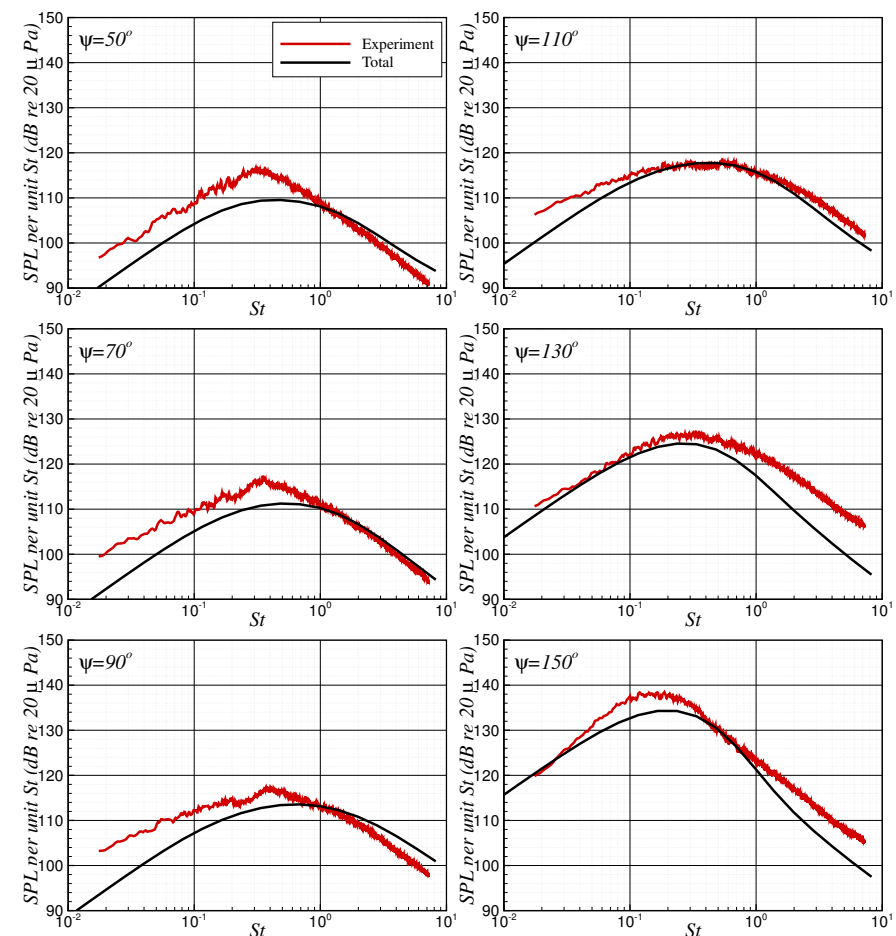

Figure 19. Comparison of predictions (Case SMC0182) with measurement of the SMC018 convergent-divergent nozzle, $M_{d}=1.80$, operating at $M_{j}=1.80$ and $T T R=1.648$ at a distance of $R / D=100$ and angles $\psi$.
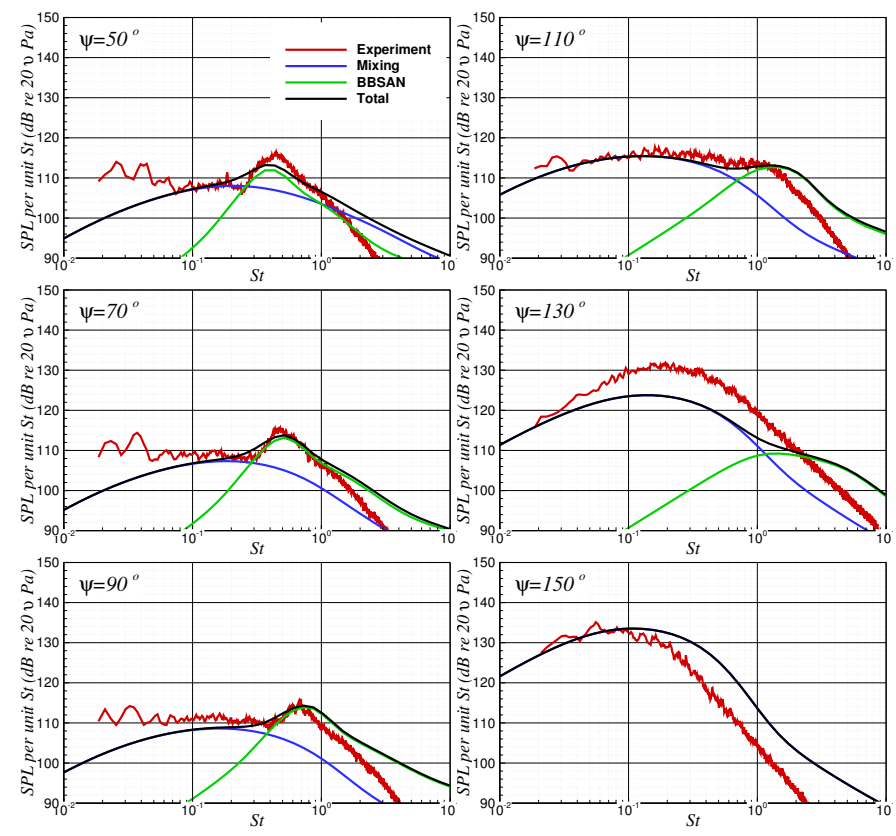

Figure 20. Comparison of predictions (Case Axi5) with measurement of the Boeing convergent dual-stream nozzle operating at $M_{j p}=1.281 \& M_{j s}=0.848$ and $T T R_{p}=2.87 \& T T R_{s}=1.00$ at a distance of $R / D=100$ and angles $\psi$. 